\title{
High-pressure behavior of structural, optical, and electronic transport properties of the golden $\mathrm{Th}_{2} \mathrm{~S}_{3}$-type $\mathrm{Ti}_{2} \mathrm{O}_{3}$
}

\author{
Sergey V. Ovsyannikov, ${ }^{1,}{ }^{*}$ Xiang Wu, ${ }^{2}$ Gaston Garbarino, ${ }^{3}$ Manuel Núñez-Regueiro, ${ }^{4}$ Vladimir V. Shchennikov, ${ }^{5}$ \\ Julia A. Khmeleva, ${ }^{5}$ Alexander E. Karkin, ${ }^{6}$ Natalia Dubrovinskaia, ${ }^{7}$ and Leonid Dubrovinsky ${ }^{1}$ \\ ${ }^{1}$ Bayerisches Geoinstitut, Universität Bayreuth, Universitätsstrasse 30, Bayreuth D-95447, Germany \\ ${ }^{2}$ School of Earth and Space Sciences, Peking University, Beijing 100871, China \\ ${ }^{3}$ European Synchrotron Radiation Facility (ESRF), 6 Rue Jules Horowitz, BP 220, F-38043 Grenoble Cedex, France \\ ${ }^{4}$ Institut Néel, Université Grenoble Alpes and Centre National de la Recherche Scientifique (CNRS), 25 Avenue des Martyrs, \\ F-38042 BP 166 Grenoble Cedex 9, France \\ ${ }^{5}$ High Pressure Group, Institute of Metal Physics, Russian Academy of Sciences, Urals Division, GSP-170, 18 S. Kovalevskaya Str., \\ Yekaterinburg 620041, Russia \\ ${ }^{6}$ Research Department on Nuclear Reactor, Institute of Metal Physics, Russian Academy of Sciences, Urals Division, GSP-170, 18 S. \\ Kovalevskaya Str., Yekaterinburg 620041, Russia \\ ${ }^{7}$ Lehrstuhl für Kristallographie, Physikalisches Institut, Universität Bayreuth, 95440 Bayreuth, Germany \\ (Received 22 January 2013; revised manuscript received 25 August 2013; published 19 November 2013)
}

\begin{abstract}
Recently, a golden colored, dense polymorph of titanium sesquioxide, $\mathrm{Ti}_{2} \mathrm{O}_{3}$ with a $\mathrm{Th}_{2} \mathrm{~S}_{3}$-type structure, has been synthesized at high-pressure high-temperature conditions. In this paper, we present results of investigations of structural, optical, and electronic transport properties of this unusual golden polymorph of $\mathrm{Ti}_{2} \mathrm{O}_{3}$ under high pressure. Several experimental techniques were used, including x-ray diffraction studies using synchrotron radiation, Raman spectroscopy, electrical resistivity, and thermoelectric power. The structural studies showed that the $\mathrm{Th}_{2} \mathrm{~S}_{3}$-type lattice is conserved under pressure, while it is subjected to an isostructural phase transition with a $\sim 0.7 \%$ volume drop at $38.5 \mathrm{GPa}$. We speculated that this transition could be driven by the $s \rightarrow d$ electron transfer in the Ti atoms. For the $\mathrm{Th}_{2} \mathrm{~S}_{3}$-type $\mathrm{Ti}_{2} \mathrm{O}_{3}$, we have established a bulk modulus value, $B_{0}=258.3 \mathrm{GPa}$ at $B_{0}^{\prime}=4.1$. A full profile analysis of the diffraction patterns allowed us to discover anomalies in the compression behavior of the $\mathrm{Th}_{2} \mathrm{~S}_{3}$-type structure. The bond valence sums method suggested that at ambient conditions the Ti cations have predominantly $\mathrm{Ti}^{3+}$ oxidation state, but applied pressure stimulates a partial charge disproportionation between the Ti1 and Ti2 sites achieving the maximal effect-reduction of the Til cations to $\sim \mathrm{Ti}^{2.5+}$ and oxidation of the Ti2 ones to $\sim \mathrm{Ti}^{3.5+}$ near $14 \mathrm{GPa}$. Pressure evolution of Raman spectra across the above crossovers showed distinct changes corroborating the above findings. The high-pressure electronic transport studies confirmed that the $\mathrm{Th}_{2} \mathrm{~S}_{3}$-type $\mathrm{Ti}_{2} \mathrm{O}_{3}$ remains semiconducting up to $21 \mathrm{GPa}$ at ambient and low temperatures down to $4.2 \mathrm{~K}$. These studies found additional features, e.g., in the activation energy curve near $7 \mathrm{GPa}$, that is accompanied by inversion of the dominant conductivity type from electron to hole. The intriguing high-pressure behavior of $\mathrm{Ti}_{2} \mathrm{O}_{3}$ with the $\mathrm{Th}_{2} \mathrm{~S}_{3}$-type structure can contribute to better understanding of high-pressure properties of transition-metal sesquioxides.
\end{abstract}

DOI: 10.1103/PhysRevB.88.184106

PACS number(s): 72.20.Pa, 73.61.Ey, 71.22.+i, 61.50.Ks

\section{INTRODUCTION}

Oxide materials find numerous industrial applications. This fact is related to both relatively simple and low-cost methods of their preparation and their unique properties. One of the industry-oriented subclasses of oxides is sesquioxides, $M_{2} \mathrm{O}_{3}$, where $M$ is a metal or combination of metals. Several well-known multifunctional families of industrial materials for magnetic, piezoelectric, and other applications belong to this class, e.g., manganites $\left(M \mathrm{MnO}_{3}\right)$, ferrites $\left(M \mathrm{FeO}_{3}\right)$, and titanates $\left(M \mathrm{TiO}_{3}\right)$. This explains the enormous interest in this class of oxides and intensive investigations of its representatives. Binary sesquioxides are parent compounds for ternary and quaternary sesquioxides; hence, their properties partly predetermine those that may be achieved in multication systems. Therefore, a better understanding of binary systems can help in the design of new oxide materials with desirable properties and, in general, would stimulate further advances in the field.

At ambient conditions, the binary sesquioxides, $M_{2} \mathrm{O}_{3}$, seem to be rather monotonous systems that show a tendency to crystallization into two simple crystal lattices, namely (i) a rhombohedral corundum lattice (space group \#167, $R \overline{3} c$ ) in systems with the lighter cations (e.g., Al, Fe, Cr, V, and Ti) and (ii) a cubic bixbyite lattice (space group \#206, I $\bar{a} 3$ ) in systems with the heavier cations (e.g., Y, In, La, Pr, Nd, Sm, $\mathrm{Eu}, \mathrm{Gd}, \mathrm{Dy}, \mathrm{Er}, \mathrm{Tm}, \mathrm{Yb}, \mathrm{Sc}, \mathrm{Mn}){ }^{1}$ To complete this picture, notice that these two groups are a bit overlapped (e.g., the cases of Sc and $\mathrm{Mn}$ oxides), and there are several exceptions: for instance, $\mathrm{Co}_{2} \mathrm{O}_{3}, \mathrm{Rb}_{2} \mathrm{O}_{3}$, and $\mathrm{Sb}_{2} \mathrm{O}_{3}$ adopt cubic lattices with space groups $F d \overline{3} m Z$ (\#227; Ref. 2), I $\overline{4} 3 d$ (\#220; Ref. 3), and $F d \overline{3} m$ (\#227; Ref. 4), respectively; and $\mathrm{Ga}_{2} \mathrm{O}_{3}, \mathrm{Bi}_{2} \mathrm{O}_{3}$, and $\mathrm{Ni}_{2} \mathrm{O}_{3}$ adopt monoclinic lattices with space groups $C 2 / \mathrm{m}$ (\#12; Ref. 5) and $P 22_{1} / c$ (\#14; Ref. 6) and a hexagonal lattice with space group $P 6_{3} m c$ (\#186; Ref. 7), respectively.

Under application of high pressure, the sesquioxides are known to undergo a series of phase transitions. However, with a few exceptions, the pressure-temperature phase diagrams of sesquioxides remain poorly explored. The most studied systems with the corundum-type lattice, such as $\mathrm{Al}_{2} \mathrm{O}_{3}$ and $\mathrm{Fe}_{2} \mathrm{O}_{3}$, under pressure application and heating 
to high temperatures showed transformations at first to a $\mathrm{Rh}_{2} \mathrm{O}_{3}$ (II)-type structure (space group \#60, Pbna) ${ }^{8-15}$ and then to a postperovskitelike phase of a $\mathrm{CaIrO}_{3}$-type structure (space group \#63, Cmcm), ${ }^{15-22}$ similar to a postperovskite phase originally discovered in $\mathrm{MgSiO}_{3} \cdot{ }^{23,24}$ High-pressure high-temperature (HP-HT) studies of systems crystallizing at ambient conditions in the cubic-bixbyite lattice established transitions to the corundum and then to the $\mathrm{Rh}_{2} \mathrm{O}_{3}$ (II)-type lattices (e.g., this happens in $\mathrm{In}_{2} \mathrm{O}_{3}$ ). ${ }^{25-29}$ This fact, combined with a circumstance that many other oxides subjected to HP-HT treatment, can turn into a metastable corundum lattice of which density is just slightly higher than one of the cubic-bixbyite lattice [e.g., $\mathrm{In}_{2} \mathrm{O}_{3}$ (Refs. 25, 26, and 29), $\mathrm{Ga}_{2} \mathrm{O}_{3}$ (Ref. 30), and $\mathrm{Mn}_{2} \mathrm{O}_{3}$ (Ref. 31)], could hint at a possible similarity in the phase transition scenarios under applied high pressures in the corundum, cubic-bixbyite, and other systems.

However, recent HP-HT studies suggested new trends in the phase transition scenarios of these systems. For instance, HPHT studies of cubic-bixbyite-structured $\mathrm{Mn}_{2} \mathrm{O}_{3}$ discovered a new intermediate phase ${ }^{31}$ between the corundum lattice and the postperovskite lattice of the $\mathrm{CaIrO}_{3}$-type. ${ }^{22}$ This intermediate phase adopts a triclinically distorted double perovskite lattice with three oxidation states of the $\mathrm{Mn}$ ions, such as $2+, 3+$, and $4+$ (Ref. 31). A mixture of $\mathrm{In}_{2} \mathrm{O}_{3}$ and $\mathrm{Mn}_{2} \mathrm{O}_{3}$ treated under HP-HT conditions demonstrated the formation of a class of unusual perovskite with unusual properties. ${ }^{32,33}$ Several other cubic-bixbyite-structured oxides (e.g., $\mathrm{In}_{2} \mathrm{O}_{3}, \mathrm{Sc}_{2} \mathrm{O}_{3}$, and $\mathrm{Y}_{2} \mathrm{O}_{3}$ ) under HP-HT conditions showed a transformation to a very dense and unusual oxide structure of the $\alpha-\mathrm{Gd}_{2} \mathrm{~S}_{3}$-type with Pnma space group. ${ }^{34-37}$ Peculiar scenarios of phase transitions under HP-HT conditions may be found in oxides that at ambient conditions crystallize in lattices, which are different from both the corundum and the cubic-bixbyite types, e.g., in $\mathrm{Bi}_{2} \mathrm{O}_{3}$ (Ref. 38).

Recently, a new and very dense sulfide structure of a $\mathrm{Th}_{2} \mathrm{~S}_{3}$ type (space group \#62, Pnma) ${ }^{39}$ with the coordination numbers $\mathrm{CN}=7$ and 8 has been found in $\mathrm{Ti}_{2} \mathrm{O}_{3}$ subjected to HP-HT treatment. ${ }^{40,41}$ At ambient conditions, $\mathrm{Ti}_{2} \mathrm{O}_{3}$ adopts the corundum lattice, and this polymorph is known also as Tistarite. ${ }^{42}$ It has been established that the $\mathrm{Th}_{2} \mathrm{~S}_{3}$-type polymorph of $\mathrm{Ti}_{2} \mathrm{O}_{3}$ is readily recoverable at ambient conditions and has a bright golden color, which is unusual for oxides. ${ }^{40,41}$ Recent calculations of lattice stability in $\mathrm{Al}_{2} \mathrm{O}_{3}$ have found that a $\mathrm{U}_{2} \mathrm{~S}_{3}$-type lattice that is a slight modification of the $\mathrm{Th}_{2} \mathrm{~S}_{3}$-type lattice should be stable beyond a postperovskite phase above $370 \mathrm{GPa}^{43}$ Similar calculations for another corundum-type system, namely $\mathrm{V}_{2} \mathrm{O}_{3}$, have also established a possible stability of this lattice. ${ }^{44}$ Thus, the golden high-pressure polymorph of $\mathrm{Ti}_{2} \mathrm{O}_{3}$ with the $\mathrm{Th}_{2} \mathrm{~S}_{3}$-type lattice may be considered as a very dense post-postperovskite form of sesquioxides; hence, it could help to understand properties of strongly compressed oxides. This would have profound implications, for instance, for understanding of behavior of alumina and hematite in HP-HT conditions of the Earth's interiors. Furthermore, this dense and stable golden polymorph of $\mathrm{Ti}_{2} \mathrm{O}_{3}$ could find own industrial applications.

In previous works, some of the authors have examined the crystal structure and optical and electronic transport properties of the golden $\mathrm{Ti}_{2} \mathrm{O}_{3}$ at ambient pressure. ${ }^{1,41}$ In particular, it has been established that at ambient conditions the golden $\mathrm{Ti}_{2} \mathrm{O}_{3}$ is semiconducting with a narrow band gap, $E_{g} \sim 0.1-0.2 \mathrm{eV}$, and predominant $n$-type conductivity. ${ }^{1}$ The puzzle of the golden color of this polymorph has been solved in both experimental optical and theoretical studies. ${ }^{1}$

In the present work, we performed careful experimental investigations of evolution of structural, optical, and electronic transport properties of the golden $\mathrm{Ti}_{2} \mathrm{O}_{3}$ under application of high pressures up to 57,67 , and $21 \mathrm{GPa}$, respectively. With high accuracy, we have established an equation of state (EOS) of the golden $\mathrm{Ti}_{2} \mathrm{O}_{3}$. We find evidences that above 5-8 GPa, the $\mathrm{Th}_{2} \mathrm{~S}_{3}$-type $\mathrm{Ti}_{2} \mathrm{O}_{3}$ turns to a different electronic state with even narrower band gap and $p$-type conductivity. The x-ray diffraction (XRD) and Raman spectroscopy data unambiguously indicate unusual features at higher pressures, near 15-20 and 38-42 GPa. The underlying physics of those is also discussed.

\section{DETAILS OF EXPERIMENTS}

\section{A. Preparation and characterization of the $\mathrm{Th}_{2} \mathrm{~S}_{3}$-type polymorph of $\mathrm{Ti}_{2} \mathrm{O}_{3}$}

We have prepared the samples of $\mathrm{Ti}_{2} \mathrm{O}_{3}$ with the $\mathrm{Th}_{2} \mathrm{~S}_{3}$ type structure from a commercial fine powder of corundumstructured $\mathrm{Ti}_{2} \mathrm{O}_{3}$ (99.8\% purity, Alfa Aesar Company) by means of HP-HT synthesis at 1200- and 5000-tonne multianvil presses at the Bayerisches Geoinstitut (BGI, University of Bayreuth, Germany). ${ }^{30,45}$ Details of the synthesis procedures were reported in our previous papers. ${ }^{1,41}$ In this work, we investigated two samples that have been synthesized in different HP-HT conditions: sample A was compressed to $17 \mathrm{GPa}$ and was synthesized at $1000^{\circ} \mathrm{C}$ over 2 hours; likewise, sample B was compressed to $22 \mathrm{GPa}$ and was synthesized at $1800^{\circ} \mathrm{C}$ over 3 hours. We have characterized the samples at ambient pressure using scanning electron microscopy and microprobe analyses; XRD; Raman and infrared spectroscopy; and measurements of electrical resistivity, magnetoresistance, Hall effect, and thermoelectric power. ${ }^{1}$ In particular, we have confirmed the $\mathrm{Th}_{2} \mathrm{~S}_{3}$-type structure and found its unit cell parameters as follows: $a=7.8248(6) \AA, b=2.8507$ (4) $\AA$, and $c=8.0967(3) \AA$ (Ref. 1). Electronic transport studies showed the difference between samples A and B: the former was found to be an intrinsic semiconductor with a band gap $E_{g} \sim 0.2 \mathrm{eV}$ and $n$-type electrical conductivity, but the latter showed a smaller band gap, $E_{g} \sim 0.1 \mathrm{eV}$, and inhomogeneous domain structure with regions of dominating $n$ - and $p$-types of conductivity. ${ }^{1}$

\section{B. Structural and optical studies at high pressure}

For structural and optical investigations under high pressure, we used conventional diamond anvil cells with culets of $250 \mu \mathrm{m}$ and rhenium gaskets. In these experiments, we used a neon pressure-transmitting medium and loaded the cells using a gas-loading apparatus at BGI. ${ }^{46}$ XRD and Raman spectroscopy studies were performed under pressures between ambient and up to 56 and to $65 \mathrm{GPa}$, respectively. In both cases, the pressure values were determined by the shift of the ruby luminescence line. ${ }^{47}$ These studies were performed on sample A. High-resolution XRD patterns were collected at 
the ID09a line $(\lambda=0.4147 \AA)$ of the European Synchrotron Radiation Facility (ESRF; Grenoble, France). A beam was focused on a sample in a spot 4-5 $\mu \mathrm{m}$ in diameter. Data were gathered with a MAR image plate detector. Two-dimensional XRD images were integrated using a Fit2D program ${ }^{48}$ and was further analyzed by means of a full-profile Rietveld refinement method. ${ }^{49}$

Each Raman spectrum was obtained by averaging 2 to 4 spectra acquired during 5-10 minutes. The Raman spectra were excited with the red 632.8-nm line of a He-Ne laser and were recorded using a LabRam spectrometer in a backscattering geometry. ${ }^{41}$

\section{Electronic transport studies under high pressure at ambient and low temperatures}

Investigations of thermoelectric and electrical properties at high pressure and ambient temperature were carried out in two anvil-type cells with flat and semispherically concave anvils operating for measurements of thin disk-shaped samples with typical sizes of $\sim 200 \times 200 \times 30 \mu \mathrm{m}^{3}$ up to $\sim 15-30 \mathrm{GPa}$, and thick samples with typical sizes of $\sim 200 \times 200 \times 250 \mu \mathrm{m}^{3}$ up to $\sim 8-12 \mathrm{GPa}$, respectively. ${ }^{50-53}$ A sample container made of the lithographic stone (basically $\mathrm{CaCO}_{3}$ ) served both as a gasket and a pressure-transmitting medium. ${ }^{54-56}$ The experiments were carried out on an automated minipress setup with motor-driven plungers, allowing us to tune applied stress continuously and to measure several parameters of a sample and environment simultaneously upon compression and decompression cycles. ${ }^{57}$ Two Pt-Ag ribbons $5 \mu \mathrm{m}$ thick were used as electrical leads to the sample. A pressure dependence of electrical resistivity was measured by a quasi-four-probe technique (the ends of these probes were bifurcated outside the cell). ${ }^{58,59}$ In the thermopower measurements, the upper anvil was heated to generate a temperature difference, $\Delta T$, along the sample thickness. The experiments were performed in three regimes: at fixed $\Delta T$ under gradual variation in applied pressure $(P)$, at fixed $P$ and gradual variation in $\Delta T$ value, and under monotonic variations in both $\Delta T$ and $P$. All the three regimes gave similar results. Other details of the thermopower measurements can be found elsewhere. ${ }^{60}$

For low-temperature electrical resistivity studies under pressure, we used a Bridgman-type high-pressure apparatus with sintered diamond anvils. A pyrophillite gasket and two steatite disks were used as a pressure-transmitting medium. We performed four-contact electrical resistivity measurements using a Keithley 2400 current source meter and a Keithley 2182 nanovoltmeter. More details are available elsewhere. ${ }^{61}$ These studies covered a temperature range between 4.2 and $300 \mathrm{~K}$ and a pressure range between 1 and $21 \mathrm{GPa}$.

\section{RESULTS AND DISCUSSION}

We present a set of experimental results and discussion in paragraphs III.A, B, and C, focusing on pressure effects on the crystal structure, lattice dynamics, and on the electronic transport in the $\mathrm{Th}_{2} \mathrm{~S}_{3}$-type $\mathrm{Ti}_{2} \mathrm{O}_{3}$, respectively.

\section{A. XRD studies of the $\mathrm{Th}_{2} \mathrm{~S}_{3}$-type $\mathrm{Ti}_{2} \mathrm{O}_{3}$ under high pressure to $56 \mathrm{GPa}$}

A detailed analysis of the $\mathrm{Th}_{2} \mathrm{~S}_{3}$-type crystal lattice of $\mathrm{Ti}_{2} \mathrm{O}_{3}$ at ambient conditions was performed in earlier works, including those of some of the authors. ${ }^{1,40,41}$ A summary of the unit cell parameters of the $\mathrm{Th}_{2} \mathrm{~S}_{3}$-type $\mathrm{Ti}_{2} \mathrm{O}_{3}$ is given in Table I. Previous high-pressure works on the $\mathrm{Th}_{2} \mathrm{~S}_{3}$-type $\mathrm{Ti}_{2} \mathrm{O}_{3}$ indicated no dramatic phase transitions and thereby suggested that this polymorph remains stable up to at least $74 \mathrm{GPa}$ at ambient and high temperatures up to $2200{ }^{\circ} \mathrm{C} .{ }^{41}$ In this work, we performed high-quality XRD studies of the $\mathrm{Th}_{2} \mathrm{~S}_{3}$-type $\mathrm{Ti}_{2} \mathrm{O}_{3}$ under pressure up to $56 \mathrm{GPa}$ (Fig. 1). Full-profile Rietveld refinement of these XRD patterns allowed us to detect structural features.

In Fig. 1(a), one can see that applied pressure does not substantially change the XRD patterns, suggesting the persistence of the original structure, in agreement with previous works. ${ }^{1,40,41}$ In all of the XRD patterns, we detected strong reflections from both the single-crystalline diamond anvils

TABLE I. Parameters of the unit cell of the golden phase of $\mathrm{Ti}_{2} \mathrm{O}_{3}$ at ambient pressure.

\begin{tabular}{|c|c|c|c|c|c|}
\hline \multirow{2}{*}{$\begin{array}{l}\text { Unit cell: } \\
\text { Atomic coordinates: }\end{array}$} & \multicolumn{5}{|c|}{$\begin{array}{l}\text { space group \#62, Pnma, structural type: } \mathrm{Th}_{2} \mathrm{~S}_{3} \text {; lattice parameters: } a=7.8248(6) \AA \text {, } \\
\qquad b=2.8507(4) \AA \text {, and } c=8.0967(3) \AA, V=180.61(1) \AA^{3}, Z=4\end{array}$} \\
\hline & $x$ & & $y$ & $z$ & \\
\hline Ti1 & 0.0279 & & $\frac{1}{4}$ & 0.3165 & \\
\hline Ti2 & 0.1952 & & $\frac{1}{4}$ & 0.9822 & \\
\hline $\mathrm{O} 1$ & 0.9432 & & $\frac{1}{4}$ & 0.8704 & \\
\hline $\mathrm{O} 2$ & 0.1237 & & $\frac{1}{4}$ & 0.5545 & \\
\hline $\mathrm{O} 3$ & 0.7901 & & $\frac{1}{4}$ & 0.1885 & \\
\hline \multicolumn{3}{|c|}{ Ti1-O distances (in $\AA$ ) } & \multicolumn{3}{|c|}{ Ti2-O distances (in $\AA$ ) } \\
\hline Ti1-O1 & 2.091(1) & $\times 2$ & Ti2-O1 & $2.169(7)$ & \\
\hline Ti1-O2 & $2.067(7)$ & & Ti2-O1 & $2.151(5)$ & $\times 2$ \\
\hline Ti1-O2 & $2.128(3)$ & $\times 2$ & Ti2-O2 & $2.093(4)$ & $\times 2$ \\
\hline Ti1-O3 & $2.052(1)$ & & Ti2-O3 & $2.767(7)$ & \\
\hline Ti1-O3 & $2.129(9)$ & & Ti2-O3 & $1.988(7)$ & $\times 2$ \\
\hline$\langle\mathrm{Ti} 1-\mathrm{O}\rangle$ & 2.098 & & $\langle\mathrm{Ti} 2-\mathrm{O}\rangle$ & 2.176 & \\
\hline
\end{tabular}



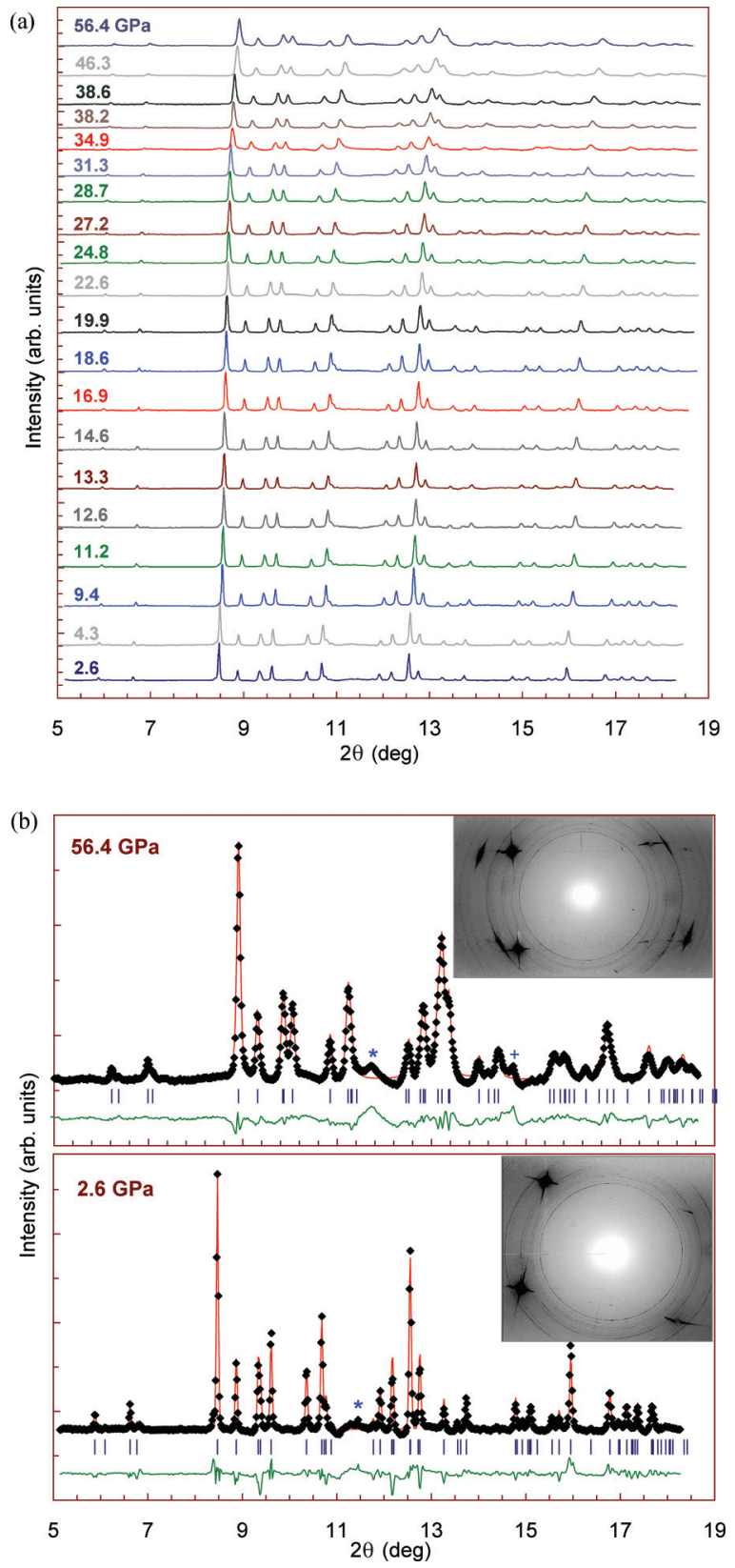

FIG. 1. (Color online) X-ray diffraction patterns of the golden $\mathrm{Th}_{2} \mathrm{~S}_{3}$-type $\mathrm{Ti}_{2} \mathrm{O}_{3}$ at ambient temperature under high pressure. (a) Pressure evolution of the XRD patterns with subtracted background. Pressure values are given near the curves. (b) Examples of Rietveld refinement of the XRD patterns collected at 2.6 and 56.4 GPa in the $\mathrm{Th}_{2} \mathrm{~S}_{3}$-type lattice (Pnma space group). Points are experimental data, solid lines are calculated profiles, dashes denote expected reflex positions for the $\mathrm{Th}_{2} \mathrm{~S}_{3}$-type lattice, and the lowermost curves are the difference between the experimental and the calculated profiles. The asterisks $(*)$ mark the residual broad peak from the diamond anvils and the cross $(+)$ in the XRD pattern (a) marks some artifact peak. Insets show the corresponding $2 \mathrm{D}$ diffraction images. The diffractions' rings are reflections from the sample, and huge spots are from the diamond anvils.

and, above $10 \mathrm{GPa}$, from the neon pressure-transmitting medium [Fig. 1(b)]. We have masked these parasite peaks using Fit2D software ${ }^{48}$ before integration of the patterns, but some remains of them still may be noticed, e.g., between $11^{\circ}$ and $12^{\circ}$ of $2 \theta$ (diamond reflections) and between $14.3^{\circ}$ and $15.3^{\circ}$ of $2 \theta$ (Ne reflections; Fig. 1). The XRD patterns of the $\mathrm{Th}_{2} \mathrm{~S}_{3}$-type $\mathrm{Ti}_{2} \mathrm{O}_{3}$ distinctly showed all the rings (about 20) corresponding to the main reflexes [insets in Fig. 1(b)]; therefore, one could apply the full-profile Rietveld refinement method $^{49}$ to figure out fine details of the crystal structure variation under compression. The Rietveld refinement analysis of all the XRD patterns confirmed the $\mathrm{Th}_{2} \mathrm{~S}_{3}$-type structure [examples in Fig. 1(b)]. The quality of these fittings was satisfactorily good with typical factors $w R p<1-2 \%$ for the original data with a background and $w R p<4-6 \%$ after the background subtraction. Under compression to $56.4 \mathrm{GPa}$, the unit cell parameters, $a, b$, and $c$ shrank by $\sim 4.7 \%, 5 \%$, and $5.7 \%$, respectively [inset in Fig. 2(a); the numerical data are available in the Supplemental Material ${ }^{62}$ ], indicating an apparent anisotropy in the axes' compressibilities. The type of lattice distortion is also conserved in the entire pressure range, i.e., $c>a$ [Fig. 2(b)], in contrast to $a>c$ for the $\mathrm{U}_{2} \mathrm{~S}_{3}$-type structures. ${ }^{41}$ A moderate contraction in the unit cell volume $\left(V_{0}\right)$ by only $\sim 14.6 \%$ to $56.4 \mathrm{GPa}$ [Fig. 2(c)] indicates a high bulk modulus value, $B_{0}$. All the unit cell parameters show an apparent discontinuity drop near $38.5 \mathrm{GPa}$ (Fig. 2). A $c / a$ ratio exhibits a distinct rising below $2.5 \mathrm{GPa}$, a drop near $38.5 \mathrm{GPa}$, and a weak inflection point near about $15 \mathrm{GPa}$ [Fig. 2(b)].

Fitting the well-known third-order Birch-Murnaghan EOS as follows:

$$
\begin{aligned}
P(V)= & \frac{3 B_{0}}{2}\left[\left(\frac{V_{0}}{V}\right)^{\frac{7}{3}}-\left(\frac{V_{0}}{V}\right)^{\frac{5}{3}}\right] \\
& \times\left\{1+\frac{3}{4}\left(B_{0}^{\prime}-4\right)\left[\left(\frac{V_{0}}{V}\right)^{\frac{2}{3}}-1\right]\right\},
\end{aligned}
$$

to the volume $(V)$ vs pressure $(P)$ data below $38.5 \mathrm{GPa}$ [Fig. 2(c)] for fixed $B_{0}^{\prime}=4$, we find $V_{0}=181.1 \AA^{3}$ and $B_{0}=$ $259.3 \mathrm{GPa}$. Freeing $B_{0}^{\prime}$ at fixed $V_{0}$, we find $B_{0}=258.3 \mathrm{GPa}$ and $B_{0}^{\prime}=4.1$. These values are in good agreement with those predicted in calculations $V_{0}=184.3 \AA^{3}$ and $B_{0}=251 \mathrm{GPa}$ at $B_{0}^{\prime}=4.5$ (Ref. 41). Fitting the entire data range would give $B_{0}=262.3 \mathrm{GPa}$ and $B_{0}^{\prime}=3.5(5)$ [Fig. 2(c)], but the quality of this fitting is apparently worse than in the above fittings limited to $38 \mathrm{GPa}$. Previous work using a LiF pressure-transmitting medium established a higher bulk modulus value, $B_{0}=296$ $\mathrm{GPa}$ at fixed $B_{0}^{\prime}=4$ (Ref. 40). In this XRD study, we used the neon pressure-transmitting medium; thus, we believe that our estimation of $B_{0}$ is more precise and not overestimated due to stresses and strains. Using the Murnaghan EOS for axial moduli determination, $P=\frac{B_{d_{0}}}{B_{d_{0}}^{\prime}}\left[\left(\frac{d_{0}^{3}}{d^{3}}\right)^{B_{d_{0}}^{\prime}}-1\right]$, where $d_{0}$ is the lattice parameter along the $d$ axis at ambient pressure, $B_{d}$ is the compression modulus of this axis, and $B_{d}^{\prime}$ is its pressure derivative fixed to 4 , we derive $a_{0}=7.823 \AA$ and $B_{a}=293 \mathrm{GPa}$, $b_{0}=2.8509 \AA$ and $B_{b}=272 \mathrm{GPa}$, and $c_{0}=8.117 \AA$ and $B_{c}=$ $221 \mathrm{GPa}$.

The full-profile Rietveld refinement of the XRD patterns of the $\mathrm{Th}_{2} \mathrm{~S}_{3}$-type $\mathrm{Ti}_{2} \mathrm{O}_{3}$ allowed us to grasp the pressure effect on the coordination spheres of the titanium ions in two different crystallographic positions, Ti1 and Ti2, with the coordination numbers, $\mathrm{CN}=7$ and 8 , respectively [insets in Figs. 2(c) and 3(b)]. We analyzed the individual [Fig. 3(a)] 

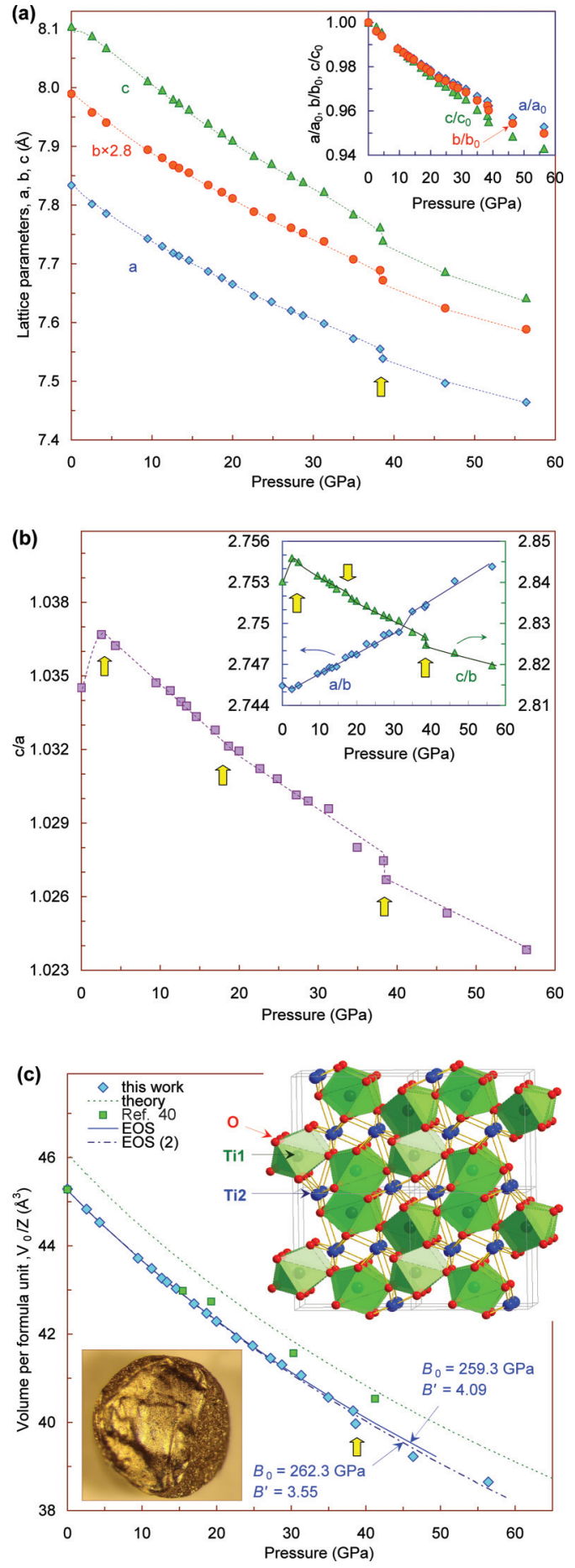

FIG. 2. (Color online) Pressure dependencies of the unit cell parameters of the golden $\mathrm{Th}_{2} \mathrm{~S}_{3}$-type $\mathrm{Ti}_{2} \mathrm{O}_{3}$ at ambient temperature. Large yellow arrows indicate the features. (a) Pressure dependencies of the lattice parameters. Inset shows relative changes of the lattice parameters with pressure. (b) Pressure dependencies of the lattice parameter ratios, $c / a$ (main plot), $a / b$ and $c / b$ (inset). (c) Pressure dependence of the unit cell volume per formula unit $\left(V_{0} / Z\right)$. Points are the experimental data; solid and dashed lines are fittings and theoretical prediction. The error bars are smaller than the symbols. Photograph of the golden polymorph of $\mathrm{Ti}_{2} \mathrm{O}_{3}$ and its crystal structure in the form of a $2 \times 2 \times 2$ supercell are shown as lower and upper insets, respectively. and average bond lengths [Fig. 3(b)], the polyhedra volumes [Fig. 3(c)] and distortion indexes [Fig. 3(d)], and the bond valence sums (BVS) of the cations [Fig. 3(e)]. This analysis revealed interesting features in all of the aforementioned parameters under pressure. For instance, one can notice that under pressurization up to $\sim 15 \mathrm{GPa}$, the average $\langle\mathrm{Ti} 2-\mathrm{O}\rangle$ length is strongly contracted, but the average $\langle\mathrm{Ti1}-\mathrm{O}\rangle$ length on the contrary remains approximately the same [Fig. 3(b)]. The unusual behavior of the $\langle\mathrm{Ti1}-\mathrm{O}\rangle$ length is related to the extension of the T1-O2 bonds and reduction in the Ti1-O1 and Ti1-O3 bonds [Fig. 3(a)]. This points out strong distortions of the Ti1 and Ti2 polyhedra under pressure; hence, we calculated their distortion index $\delta$ as follows: ${ }^{63}$

$$
\delta=\frac{\sqrt{\frac{1}{\mathrm{CN}} \sum_{i=\mathrm{CN}}\left[\left(l_{\mathrm{Ti}-\mathrm{O}}\right)_{i}-\left\langle l_{\mathrm{Ti-O}}\right\rangle\right]^{2}}}{\left\langle l_{\mathrm{Ti}-\mathrm{O}}\right\rangle},
$$

where $\mathrm{CN}$ is the coordination number, and $\left(l_{\mathrm{Ti}-\mathrm{O}}\right)_{i}$ and $\left\langle l_{\mathrm{Ti}-\mathrm{O}}\right\rangle$ are the individual and average bond lengths, respectively. We find that at ambient pressure the distortion index of the Ti1 polyhedra was minimal, but with pressure it rises by a factor of about 3 [Fig. 3(d)]. The Ti2 polyhedra are already strongly distorted at ambient pressure, and their distortion index fluctuates around $\delta \sim 0.10-0.13$ in the entire pressure range [Fig. 3(d)].

Pressure variations in the individual Ti1-O2 and all the Ti2$\mathrm{O}$ bonds demonstrate the apparent features around 15-20 GPa [Fig. 3(a)]. The pressure curves of the average bond lengths, $\langle\mathrm{Ti} 1-\mathrm{O}\rangle$ and $\langle\mathrm{Ti} 2-\mathrm{O}\rangle$, converge to about $15 \mathrm{GPa}$ and diverge on further pressurization [Fig. 3(b)]. The strongest distortion of the Ti2 polyhedra is achieved at pressures around $15 \mathrm{GPa}$ [Fig. 3(d)]; at these pressure values the Ti1 polyhedra also show a bend in the distortion index. The pressure curves of the polyhedra volumes exhibit the distinct kinks at the same pressures [Fig. 3(c)]. The significant difference in the pressure evolution of the coordination spheres of the Ti1 and Ti 2 cations might be related to changes in the oxidation states of the Ti1 and Ti2 ions under pressure and their deviations from the trivalent state. Titanium ions are known to be able to show $4+$ and $2+$ oxidation states (e.g., in $\mathrm{TiO}_{2}, \mathrm{TiO}$ ); hence, they could also show up in the $\mathrm{Th}_{2} \mathrm{~S}_{3}$-type $\mathrm{Ti}_{2} \mathrm{O}_{3}$ in the course of the charge disproportionation reaction between the $\mathrm{Ti}^{3+}$ cations. It is worth mentioning here that theoretical calculations predicted the instability of the $\mathrm{Th}_{2} \mathrm{~S}_{3}$-type $\mathrm{Ti}_{2} \mathrm{O}_{3}$ against decomposition into $\mathrm{TiO}_{2}$ and $\mathrm{TiO}$ at high pressures above $150 \mathrm{GPa}^{41}$

As the oxidation states should affect the effective ionic radii of the Ti ions, in order to estimate the oxidation states of the Ti1 and Ti2 ions, we applied the BVS method, in which the bond valence $\left(S_{i j}\right)$ and their sum $\left(V_{i}\right)$ come as follows: ${ }^{64}$

$$
s_{i j}=\exp \left[\frac{R_{i j}-d_{i j}}{b_{0}}\right] ; \quad V_{i}=\sum_{j} s_{i j},
$$

where $d_{i j}$ is the distance between the atoms $i$ and $j, R_{i j}$ is the empirically determined distance for this cation-anion pair, and $b_{0}$ is an empirical parameter that is usually about 0.37 . But in the case of the high-pressure polymorph of $\mathrm{Ti}_{2} \mathrm{O}_{3}$, which is denser by $10-12 \%$ than the corundum-type $\mathrm{Ti}_{2} \mathrm{O}_{3}$ (Refs. 1, 40, and 41), the parameter $b_{0}$ should be accordingly smaller than 0.37. In general, application of the BVS method for tracking changes under pressure or temperature variation 

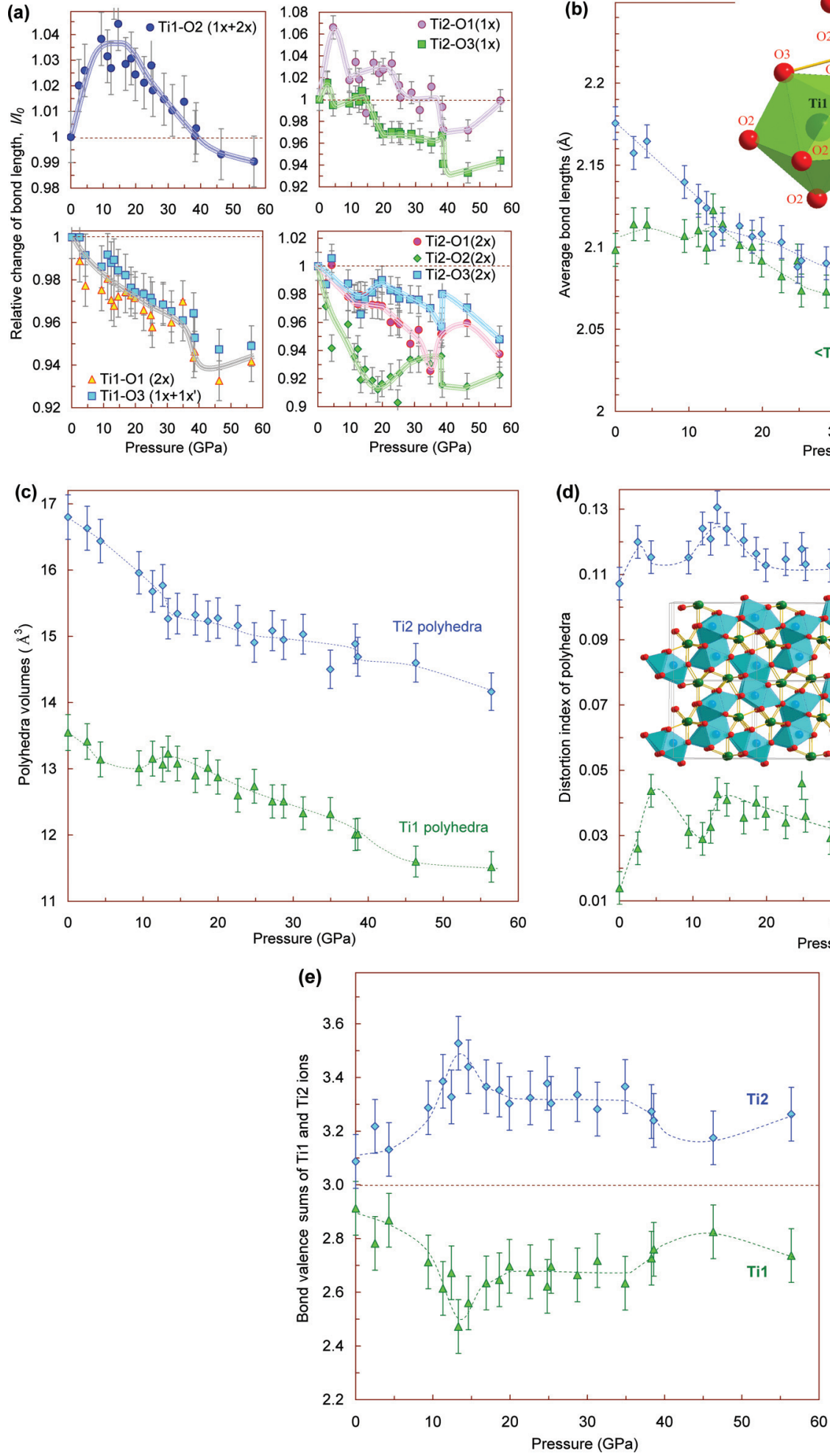

FIG. 3. (Color online) Pressure dependencies of the bond lengths, polyhedra volumes and distortion indexes, and bond valence sums of the Ti ions in the $\mathrm{Th}_{2} \mathrm{~S}_{3}$-type $\mathrm{Ti}_{2} \mathrm{O}_{3}$ at ambient temperature. (a) Relative changes in the bond lengths with pressure. Labels $1 \times$ and $2 \times$ denote the multiplicity of the bond lengths. Some of the curves correspond not to the individual bond lengths but to their combinations if these bonds demonstrate rather similar behavior under pressure [e.g., Ti1-O2 $(1 \times$ and $2 \times)$ is a combination of three Ti1-O2 bonds, two of which are identical (Table I)]. The pressure behavior of all the bonds demonstrates a number of features. (b) Pressure dependencies of the average bond lengths, $\langle\mathrm{Ti} 1-\mathrm{O}\rangle$ and $\langle\mathrm{Ti} 2-\mathrm{O}\rangle$. Inset shows the coordination spheres of the Ti1 and Ti2 ions in the $\mathrm{Th}_{2} \mathrm{~S}_{3}$-type structure. (c) Shrinkage in the coordination polyhedra volumes of the Ti1 and T2 ions with pressure. (d) Distortion indexes of the coordination polyhedra of the Ti1 and Ti2 ions calculated using Eq. (2). Inset shows a network of the Ti2 coordination polyhedra. (e) Pressure dependencies of the bond valence sums of the Ti1 and Ti2 ions calculated using Eq. (3). Thick lines in (a) and dashed lines in (b)-(e) are guides for the eye. 
is rather limited as both the $R_{i j}$ and $b_{0}$ parameters should be functions of pressure/temperature for each cation. However, for the single-cation $\mathrm{Ti}_{2} \mathrm{O}_{3}$, the parameters $R_{i j}$ and $b_{0}$ of the $\mathrm{Ti}$ ions at the both $\mathrm{Ti} 1$ and $\mathrm{Ti} 2$ sites should be the same or very similar; hence, a relative charge disproportionation between the Ti1 and Ti2 ions may be figured out if a charge conservation under pressure is assumed. With empirical parameter $R_{i j}=1.791 \AA$ for the $\mathrm{Ti}^{3+}-\mathrm{O}$ bond, ${ }^{64}$ we find that the sum charge of the Ti1 and Ti2 ions is $6 \mathrm{e}^{+}$for the parameter $b_{0}$ equal to 0.3491 [Eq. (3)]. Using these parameters, we find that at ambient conditions, the BVS of the Ti1/Ti2 ions are $2.91+/ 3.09+$, respectively. Similarly, we calculated the BVS of the Ti1 and Ti2 ions under pressure [Fig. 3(e)]. These BVS curves demonstrate that applied pressure above roughly $5 \mathrm{GPa}$ stimulates a charge disproportionation between the cations occupying the Ti1 and Ti2 sites [Fig. 3(e)]. The maximal charge difference between the Ti1 and Ti2 ions is achieved at pressures near $14 \mathrm{GPa}$ [Fig. 3(e)], at these pressures roughly half of the $\mathrm{Ti}^{3+}$ ions at the Til sites reduce to $\mathrm{Ti}^{2+}$ and a half of the $\mathrm{Ti}^{3+}$ ions at the $\mathrm{Ti} 2$ sites oxidize to $\mathrm{Ti}^{4+}$ [Fig. 3(e)], suggesting the intricate valence formula of $\mathrm{Ti}_{2} \mathrm{O}_{3}$ as $\left[\left(\mathrm{Ti}^{2+} \mathrm{Ti}^{3+}\right)_{\text {site } 1}\right]\left[\left(\mathrm{Ti}^{3+} \mathrm{Ti}^{4+}\right)_{\text {site } 2}\right] \mathrm{O}_{6}$. Under higher pressures, the charge difference between the Ti1 and Ti2 cations are partially balanced, and the charges of the Ti1/Ti2 cations are stabilized around $2.7 \mathrm{e}^{+} / 3.3 \mathrm{e}^{+}$, respectively [Fig. 3(e)]. There are also changes in the BVS curves across the isostructural phase transition at $\sim 38.5 \mathrm{GPa}$ [Fig. 3(e)].

The ionic radii of the $\mathrm{Ti}^{2+}, \mathrm{Ti}^{3+}$, and $\mathrm{Ti}^{4+}$ cations are known to be about $\sim 1.00,0.81$, and $0.745 \AA$, respectively. Therefore, the partial reduction of the Ti1 cations under pressure to about $14 \mathrm{GPa}$ [Fig. 3(e)] should increase their average (effective) radius; this could explain the abnormal pressure dependencies of both the average $\langle$ Ti1-O $\rangle$ bond length [Fig. 3(b)] and the T1 polyhedra volume [Fig. 3(c)]. Likewise, the rapid shrinkage in both the $\langle\mathrm{Ti} 2-\mathrm{O}\rangle$ bond length [Fig. 3(b)] and the T2 polyhedra volume [Fig. 3(c)] with pressure to $14 \mathrm{GPa}$ may be explained by the partial oxidation of the Ti2 cations that enhanced the pure pressure effect. Behavior of the average bond lengths and the polyhedra volumes at higher pressures is also in good agreement with the charge disproportion reaction between the Ti1 and Ti2 cations. Notice that corundum-type $\mathrm{Ti}_{2} \mathrm{O}_{3}$ is known to display appreciable correlated effects; ${ }^{65}$ these effects might be enhanced in the denser $\mathrm{Th}_{2} \mathrm{~S}_{3}$-type polymorph, and they could affect the charge disproportion under pressure.

Such a pressure-driven charge transfer between cations sitting in different crystallographic positions was already reported for some oxides, e.g., it was documented by Mössbauer spectroscopy method in $\mathrm{FeTiO}_{3}$ (Refs. 66 and 67), by the BVS method in $\mathrm{BiNiO}_{3}$ (Ref. 68), and by structural and optical techniques in $\mathrm{Co}_{3} \mathrm{O}_{4}$ (Ref. 69). Significant charge disproportionation between different crystallographic sites can even lead to quasi-isostructural phase transitions that may be accompanied by volumetric effects and changes in lattice symmetry, e.g., this happens in double perovskites such as $\mathrm{LaCu}_{3} \mathrm{Fe}_{4} \mathrm{O}_{12}$ (Refs. 70 and 71) and $\mathrm{CaCu}_{3} \mathrm{Fe}_{4} \mathrm{O}_{12}$ (Ref. 72). However, these transitions may be so subtle that even in high-resolution powder XRD studies, it might be difficult to detect them. ${ }^{70-72}$ Thus, in the $\mathrm{Th}_{2} \mathrm{~S}_{3}$-type $\mathrm{Ti}_{2} \mathrm{O}_{3}$ we did not observe any structural transition across $14 \mathrm{GPa}$.
The tiny discontinuities in the unit cell parameters of the golden $\mathrm{Ti}_{2} \mathrm{O}_{3}$ near $38.5 \mathrm{GPa}$ (Fig. 2) have a correspondence with jumps in the pressure curves of almost all the Ti2-O bond lengths [Fig. 3(a)]. On the contrary, the Ti1-O bonds show rather smooth changes across this pressure [Fig. 3(a)]. We found that the unit cell volume near $38.5 \mathrm{GPa}$ drops by about $0.7 \%$ [Fig. 2(c)]. This volumetric effect suggests that this feature could be a first-order isostructural phase transition. However, the BVS curves show no abrupt anomalies at this pressure [Fig. 3(e)], thereby suggesting that the charge disproportionation between the Ti1 and Ti2 cations cannot be a major driving factor of this transition. Perhaps this isostructural transition is induced by variation in the electronic structure of the $\mathrm{Ti}$ atoms. It is interesting to note that elemental $\mathrm{Ti}$ metal itself in its hexagonal close-packed ( $h c p)$ phase was found to exhibit a remarkable jump in its lattice parameters ratio, $c / a$, from $\sim 1.57$ to $\sim 1.61$ at almost the same pressure value, between 36 and $44 \mathrm{GPa}^{73}$ This feature in the $c / a$ ratio was reproduced in two experiments, and, hence, is a robust finding. ${ }^{73}$ However, the nature of this feature has not been yet specified; potentially, it could be a first-order isostructural phase transition with a tiny volumetric effect. ${ }^{73}$ Earlier works reported for the Group IV elements (i.e., Ti, $\mathrm{Zr}$, and $\mathrm{Hf}$ having electronic configurations as $[\mathrm{Ar}] 4 s^{2} 3 d^{2},[\mathrm{Kr}] 5 s^{2} 4 d^{2}$, and [Xe] $6 s^{2} 4 f^{14} 5 d^{2}$, respectively) that applied pressure upshifts the $s$-band position in their electronic structures above the Fermi level. ${ }^{74}$ Therefore, at certain pressure values, the $d$ band already underlies the $s$ band, and the electronic transfer from the latter to the former becomes energetically favorable. ${ }^{74}$ Such an $s \rightarrow d$ electron transfer is assumed to lead to structural or slight isostructural transitions. ${ }^{74}$ For instance, Hf metal was found to undergo a $h c p \rightarrow \omega$-phase transition near $38 \mathrm{GPa}^{75,76}$ The case of $\mathrm{Zr}$ metal is more interesting; it shows first a series of conventional phase transitions, such as $h c p \rightarrow$ $\omega$-phase $\rightarrow$ body-centered-cubic $(b c c)$ at $\sim 6-8$ and $\sim 26 \mathrm{GPa}$, respectively, ${ }^{77-79}$ and then in the $b c c$ phase, at about $53 \mathrm{GPa}$ it undergoes an isostructural phase transition with about a $1 \%$ volume drop. ${ }^{77,78}$ This isostructural transition in $\mathrm{Zr}$ metal has been further addressed to the $s \rightarrow d$ electron transfer. ${ }^{74}$ The case of Ti metal remains poorly studied, and even the $P-T$ boundary of the $h c p \rightarrow \omega$-phase transition is still a point at issue. $^{74,75,79,80}$ Since $\mathrm{Zr}$ is the nearest electronic analogue of $\mathrm{Ti}$ in Group IV, by analogy with $\mathrm{Zr}$, one can propose that the jump in the $c / a$ ratio in Ti metal observed between 36 and $44 \mathrm{GPa}$ in earlier work ${ }^{73}$ could, in fact, be related to the previously discussed $s \rightarrow d$ electron transfer. Consequently, we can propose that the isostructural transition found in the $\mathrm{Th}_{2} \mathrm{~S}_{3}$-type $\mathrm{Ti}_{2} \mathrm{O}_{3}$ at about $38.5 \mathrm{GPa}$ (Fig. 2) may be related to this $s \rightarrow d$ electron transfer in the Ti atoms. Since the golden polymorph of $\mathrm{Ti}_{2} \mathrm{O}_{3}$ under these pressure values was found to have the Ti ions in three different oxidations states $[2+$, $3+$, and 4+; Fig. 3(e)], this $s \rightarrow d$ electron transfer should primarily affect the chemical bonds that are formed by the $s$ electrons of the $\mathrm{Ti}$ atoms (i.e., in $\mathrm{Ti}^{4+}$ and $\mathrm{Ti}^{3+}$ ions). This could explain why the changes near $38.5 \mathrm{GPa}$ in the Ti2-O bond lengths formed by the $\mathrm{Ti}^{3+}$ and $\mathrm{Ti}^{4+}$ ions are stronger than those in the Ti1-O bond lengths formed by the $\mathrm{Ti}^{3+}$ and $\mathrm{Ti}^{2+}$ ions [Figs. 3(a) and 3(e)].

The emergence of the signatures of the electronic transition in the $\mathrm{Ti}$ atoms in the high-pressure properties of the dense 
golden polymorph of $\mathrm{Ti}_{2} \mathrm{O}_{3}$ seems to be an unexpected finding. Usually, such (quasi)-isostructural transitions are driven by dramatic changes in oxidation states or by spin transitions in cations. As examples of the former, one can recall, for instance, a valence transition in $\mathrm{SmS}, \mathrm{Sm}^{2+} \rightarrow \mathrm{Sm}^{3+}+\mathrm{e}^{-}$, leading to both delocalization of electrons and isostructural transition in a NaCl-type lattice (so-called golden polymorph of $\mathrm{SmS})^{81-83}$ or charge disproportionation between different cations, e.g., between the $\mathrm{Cu}$ and $\mathrm{Fe}$ ions in $\mathrm{LaCu}_{3} \mathrm{Fe}_{4} \mathrm{O}_{12}$ (Refs. 70 and 71) and between two $\mathrm{Fe}$ ions in $\mathrm{CaCu}_{3} \mathrm{Fe}_{4} \mathrm{O}_{12}$ (Ref. 72), leading to phase transitions with changes in the lattice symmetry. Examples of the latter are well presented by spin transitions in $\mathrm{Fe}$ and $\mathrm{Co}$ ions. For instance, it was reported that a high-to-low spin transition in $\mathrm{Fe}$ ions induces an isostructural phase transition with about 5\% volume drop in $R \mathrm{FeO}_{3}(R=\mathrm{Pr}, \mathrm{Eu}, \mathrm{Lu})$ perovskites around $50 \mathrm{GPa},{ }^{84}$ as well as a high-to-low-spin transition of $\mathrm{Co}^{3+}$ ions in $\mathrm{BiCoO}_{3}$ near $3 \mathrm{GPa}$ leads to a phase transition with about $13 \%$ volume drop. ${ }^{85}$ Recalling that at ambient conditions the $\mathrm{Th}_{2} \mathrm{~S}_{3}$-type $\mathrm{Ti}_{2} \mathrm{O}_{3}$ is denser than the conventional corundum-type $\mathrm{Ti}_{2} \mathrm{O}_{3}$ by about $10-12 \%^{1,40,41}$ and is denser than the other titanium oxides, like $\mathrm{TiO}_{2}$, one can propose that this could be a reason why such a subtle transition was observable in the golden $\mathrm{Ti}_{2} \mathrm{O}_{3}$. Notice that isostructural phase transition was already reported for another titanium compound, namely, TiN adopting a NaCl-type structure, but at much lower pressure, $7-11 \mathrm{GPa}{ }^{86}$ Perhaps this transition in TiN is related to modification of the coordination spheres of the $\mathrm{Ti}$ ions because of valence or electronic transitions. Hence, to some extent, this isostructural transition in TiN might have some correspondence with the structural features we found in the $\mathrm{Th}_{2} \mathrm{~S}_{3}$-type $\mathrm{Ti}_{2} \mathrm{O}_{3}$ (Figs. 2 and 3).

\section{B. Raman spectroscopy of the golden $\mathrm{Th}_{2} \mathrm{~S}_{3}$-type $\mathrm{Ti}_{2} \mathrm{O}_{3}$ under pressure up to $67 \mathrm{GPa}$}

According to irreducible representations for the $\mathrm{Th}_{2} \mathrm{~S}_{3}$-type structure of $\mathrm{Ti}_{2} \mathrm{O}_{3}$, namely, $\Gamma=10 A_{g}+5 B_{1 g}+10 B_{2 g}+$ $5 B_{3 g}, 30$ phonon modes should be Raman active in this material. At ambient conditions in the spectral range above $150 \mathrm{~cm}^{-1}$, the golden $\mathrm{Ti}_{2} \mathrm{O}_{3}$ exhibits only 21 well-detectable modes, labeled as $A, B, C, \ldots U$ in Fig. 4(a). Since single crystalline samples of the $\mathrm{Th}_{2} \mathrm{~S}_{3}$-type $\mathrm{Ti}_{2} \mathrm{O}_{3}$ for polarized Raman study are not available, these modes cannot be assigned at the moment. In the previous work of some of the coauthors, a detailed Raman spectroscopy study on this material under pressure up to $74 \mathrm{GPa}$ has been performed. ${ }^{41}$ This study revealed several minor modifications in the Raman spectra of the golden $\mathrm{Ti}_{2} \mathrm{O}_{3}$ with pressure but could not properly address them because of very limited information on the crystal structure evolution under pressure. ${ }^{41}$ In general, the main Raman peaks kept in the entire pressure range to $74 \mathrm{GPa}$, thereby suggesting no dramatic phase transformations. ${ }^{41}$ In the present work, we revisited the Raman spectroscopy of the golden $\mathrm{Ti}_{2} \mathrm{O}_{3}$ under pressure and carefully documented changes in the spectra with pressure. We analyzed both the previous $^{41}$ and the new Raman spectroscopy data together and present in this paper a representative data set in Figs. 4 and 5.

A pressure behavior of the Raman spectra showed a number of moderate modifications (Fig. 4). The Raman spectra of the
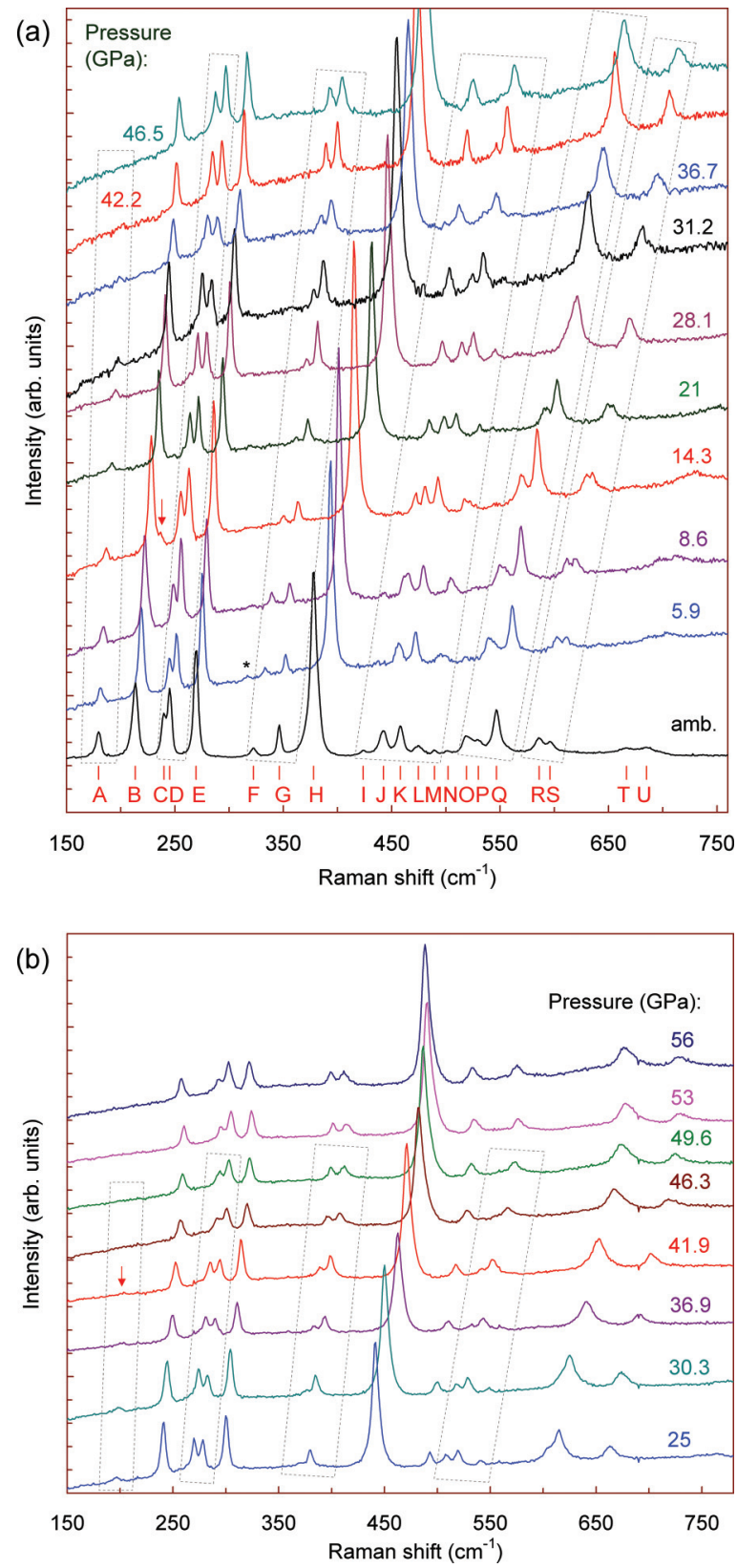

FIG. 4. (Color online) Pressure evolution of Raman spectra of the golden $\mathrm{Th}_{2} \mathrm{~S}_{3}$-type $\mathrm{Ti}_{2} \mathrm{O}_{3}$ at ambient temperature. Pressure values are given near the curves. Moderate changes in these Raman spectra are highlighted by dotted rectangles. (a) Raman spectra collected on pressurization run across the smooth crossover near $15 \mathrm{GPa}$ and the isostructural transition near $38.5 \mathrm{GPa}$ found in the XRD studies (Figs. 2 and 3). The phonon peaks in the spectrum gathered at ambient conditions are labeled by letters $A, B, \ldots U$. The asterisk marks an unclear peak. (b) Raman spectra collected on decompression run across the isostructural phase transition near $38.5 \mathrm{GPa}$ (Fig. 2).

$\mathrm{Th}_{2} \mathrm{~S}_{3}$-type $\mathrm{Ti}_{2} \mathrm{O}_{3}$ collected at ambient pressure and at $5.9 \mathrm{GPa}$ are very similar, but one can still notice that two couples of peaks, $L$ and $M$, and $O$ and $P$ around $450-550 \mathrm{~cm}^{-1}$ show a tendency to merging (Fig. 4). A further compression to $8.9 \mathrm{GPa}$ leads to splitting of the peak $J$ in the region around $450 \mathrm{~cm}^{-1}$ into a doublet (Fig. 4). With further pressurization, more noticeable features were observed that included the 

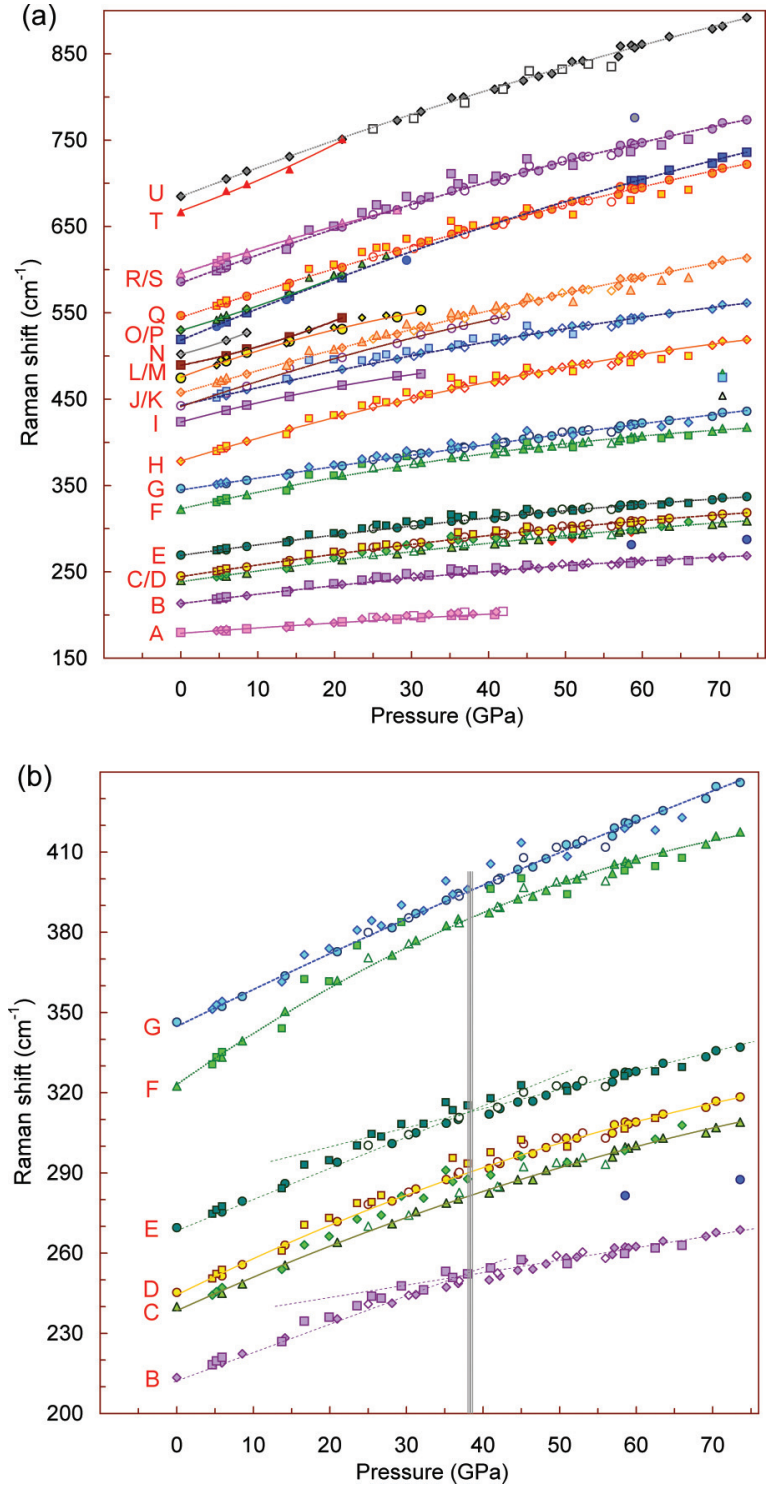

FIG. 5. (Color online) Pressure dependencies of phonon frequencies of the golden $\mathrm{Th}_{2} \mathrm{~S}_{3}$-type $\mathrm{Ti}_{2} \mathrm{O}_{3}$ at ambient temperature (summary of three sets of experiments, shown by different symbols). The phonon labels $A, B, \ldots U$ correspond to those in Fig. 4(a). (a) General view. (b) In range of wave numbers between 200 and $440 \mathrm{~cm}^{-1}$. The crossover near $38 \mathrm{GPa}$ is shown by the vertical gray line.

disappearance, appearance, merging, and splitting of different phonons. A pressure evolution of the peaks $J$ and $K$ around $450 \mathrm{~cm}^{-1}$ (at ambient pressure) revealed that to $14.3 \mathrm{GPa}$ the peak $J$ splits into a doublet, $J_{1} / J_{2}$, and under further compression the higher frequency peak of this $J_{1} / J_{2}$ doublet gradually merges with the peak $K$ (e.g., a spectrum collected at $46.5 \mathrm{GPa}$ ) [Figs. 4(a) and 5(a)]. In Fig. 4(a), we highlight by dotted rectangles the changes in peak $A$ at $\sim 180 \mathrm{~cm}^{-1}$; peaks $C$ and $D$ around $250 \mathrm{~cm}^{-1} ; F$ and $G$ around $350 \mathrm{~cm}^{-1}$; $I, J, K, L$, and $M$ around 450-500 $\mathrm{cm}^{-1} ; O, P$, and $Q$ around $550 \mathrm{~cm}^{-1}$; and $R$ and $S$ around $600 \mathrm{~cm}^{-1}$. All these changes in the Raman spectra would suggest some on-going pressure-induced processes in the crystal structure rather than a single crossover at a certain pressure value. Thus, these changes in the Raman spectra [Fig. 4(a)] seem to be compatible with the charge disproportionation reaction between the Ti1 and Ti2 cations [Fig. 3(e)] that should slightly tune the chemical bonds in the crystal lattice [Figs. 3(a) and 3(b)]. Charge disproportionation reactions in other oxides showed that besides structural anomalies, they can lead to variations in Raman spectra; this happened, e.g., in $\mathrm{Co}_{3} \mathrm{O}_{4}$ adopting a cubic spinel structure. ${ }^{69}$ Thus, the modifications in the Raman spectra we observed strongly support our above interpretation of the structural data in the terms of charge disproportionation reactions [Fig. 3(e)].

Beyond about $40 \mathrm{GPa}$, the Raman spectra did not exhibit any remarkable changes, such as appearance/disappearance and splitting/merging of the peaks [Fig. 5(a)]. A decompression cycle given in Fig. 4(b) exhibits no apparent and significant changes in the Raman spectra from 56 down to 46.3 GPa. But in the downstroke spectra collected at 41.9 and $36.9 \mathrm{GPa}$, one can already notice a number of modifications, e.g., the appearance of the weak peak $A$ near $\sim 200 \mathrm{~cm}^{-1}$, the variations in relative peak intensities of the doublets $C / D$ and $F / G$ near 280 and $380 \mathrm{~cm}^{-1}$, respectively, and the appearance of the two weak phonons near the peak $K$ in a region around $540 \mathrm{~cm}^{-1}$ [Fig. 4(b)]. A detailed consideration of the pressure dependencies of phonon frequencies allows us to detect kinks in some of them, e.g., the phonons $B$ and $E$ apparently have features around 35-40 GPa [Fig. 5(b)]. In addition, the phonons $F$ and $G$ demonstrate an anticrossing effect (consequent convergence and divergence of their frequencies) with a kink at similar pressures [Fig. 5(b)]. It is interesting to note that another couple of peaks, namely, a doublet $C / D$ at $\sim 240 \mathrm{~cm}^{-1}$ at ambient pressure [Fig. 4(a)] demonstrates the opposite behavior: frequency divergence up to profound splitting to $\sim 15 \mathrm{GPa}$ [Fig. 4(a)] and then above $\sim 40 \mathrm{GPa}$, a tendency to convergence [Figs. 4(a) and 4(b)]. Thus, the Raman spectroscopy data (Figs. 4 and 5) apparently point out a crossover near $40 \mathrm{GPa}$, consistent with the previously discussed isostructural transition near $39 \mathrm{GPa}$ (Fig. 2) that was addressed to the $s \rightarrow d$ electron transfer in the Ti atoms. No apparent hysteresis between the changes in the Raman spectra documented on pressurization and releasing runs was observed. This also agrees with the above proposed electronic nature of the compression features of the $\mathrm{Th}_{2} \mathrm{~S}_{3}$-type $\mathrm{Ti}_{2} \mathrm{O}_{3}$. It is worth mentioning here that in elemental Ti metal, a low-frequency mode at $\sim 138 \mathrm{~cm}^{-1}$ assigned to the $h c p$ phase under applied pressure behaved similarly to the phonon mode $A$ in the golden $\mathrm{Ti}_{2} \mathrm{O}_{3}$ at $\sim 180 \mathrm{~cm}^{-1}$ (Figs. 4 and 5). ${ }^{87}$ This $138 \mathrm{~cm}^{-1}$ peak in Ti started to lose its intensity above $20 \mathrm{GPa}$, became very weak above $30 \mathrm{GPa}$, and disappeared at somewhat higher pressure. ${ }^{87}$

We analyzed a pressure effect on the Raman spectra intensity for two pressurization cycles in the example of the strongest phonon mode $H$ at $378 \mathrm{~cm}^{-1}$ (perhaps it is the longitudinal optical phonon). The intensity of this phonon shows only very moderate fluctuations with pressure. In accordance to a general expression, the changes in Raman spectra intensities, $I$, under pressure are related to variation in absorption coefficient as follows: $I \sim I_{0} \times[1-\exp (-\alpha)]$, where $I_{0}$ is the integral intensity in the absence of free carriers, and $\alpha$ is the absorption coefficient. Plasmon modes in metals and doped semiconductors normally belong to the ultraviolet spectral range. Hence, these plasmon modes have energies higher than those of photons in the visible range; 
therefore, the former can screen the latter. This explains, in particular, the metallic luster of metals in the visible range. The Raman intensity data unambiguously suggest that the golden $\mathrm{Ti}_{2} \mathrm{O}_{3}$ with the band gap of $E_{g} \sim 0.1-0.2 \mathrm{eV}$ at ambient conditions ${ }^{1}$ keeps its semiconducting properties across the entire pressure range investigated. Normally, conventional narrow-gap semiconductors of Groups II-VI, IV-VI, and III-V metalize under application of much less pressure, e.g., lead chalcogenides $(\mathrm{PbTe}, \mathrm{PbSe}, \mathrm{PbS})$ with band gaps $E_{g} \sim$ $0.16-0.4 \mathrm{eV}$ at ambient conditions become metallic already at $\sim 13-21 \mathrm{GPa}^{88}$ Thus, the case of semiconducting golden $\mathrm{Ti}_{2} \mathrm{O}_{3}$ seems to be unusual. Taking into account its other remarkable characteristics, such as high mechanical and thermal stability, one can surmise that this material may be promising for various industrial applications. The extended stability of the semiconducting properties of the golden $\mathrm{Ti}_{2} \mathrm{O}_{3}$ under pressure points out the rigidity of its electronic bands. This correlates with the high bulk modulus value of $\mathrm{Th}_{2} \mathrm{~S}_{3}$-type $\mathrm{Ti}_{2} \mathrm{O}_{3}, B_{0}=258.3 \mathrm{GPa}$ at $B_{0}^{\prime}=4.1$ found above [Fig. 2(c)].

\section{Electronic transport of the golden $\mathrm{Th}_{2} \mathrm{~S}_{3}$-type $\mathrm{Ti}_{2} \mathrm{O}_{3}$ under pressure up to $21 \mathrm{GPa}$}

To examine the variation in the electronic band structure parameters of the golden $\mathrm{Ti}_{2} \mathrm{O}_{3}$ under pressure, we performed two sorts of studies in different high-pressure cells. In one of those, we probed the electrical conductivity and thermoelectric power at room temperature under pressure up to $18 \mathrm{GPa}$ (Figs. 6-8). In the other study, we investigated temperature

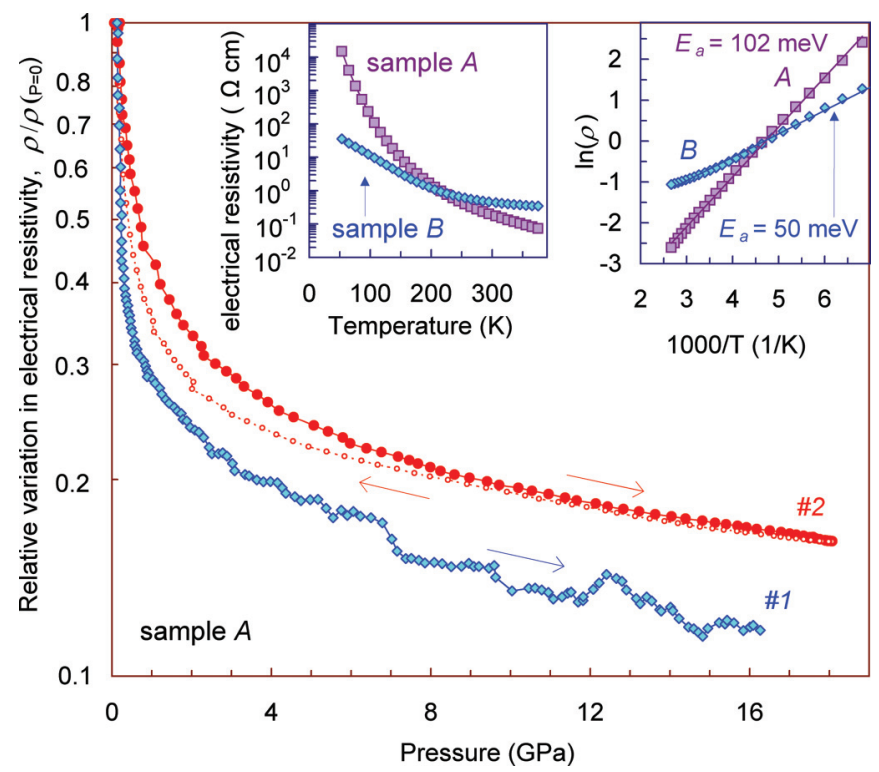

FIG. 6. (Color online) Pressure dependencies of electrical resistance of bulk sample $A$ of the golden $\mathrm{Th}_{2} \mathrm{~S}_{3}$-type $\mathrm{Ti}_{2} \mathrm{O}_{3}$ at ambient temperature. These curves were obtained in high-pressure cells with conventional flat anvils. Labels \#1 and \#2 mark two different samples cut from the same bulk sample $A$. Arrows show directions of pressure variation. Left inset shows temperature dependencies of electrical resistivity for samples $A$ and $B$ at ambient pressure. Right inset presents evidence of activation character of electrical resistivity near room temperature and down to $\sim 150 \mathrm{~K}$ and reports the activation energies values, $E_{a}$.
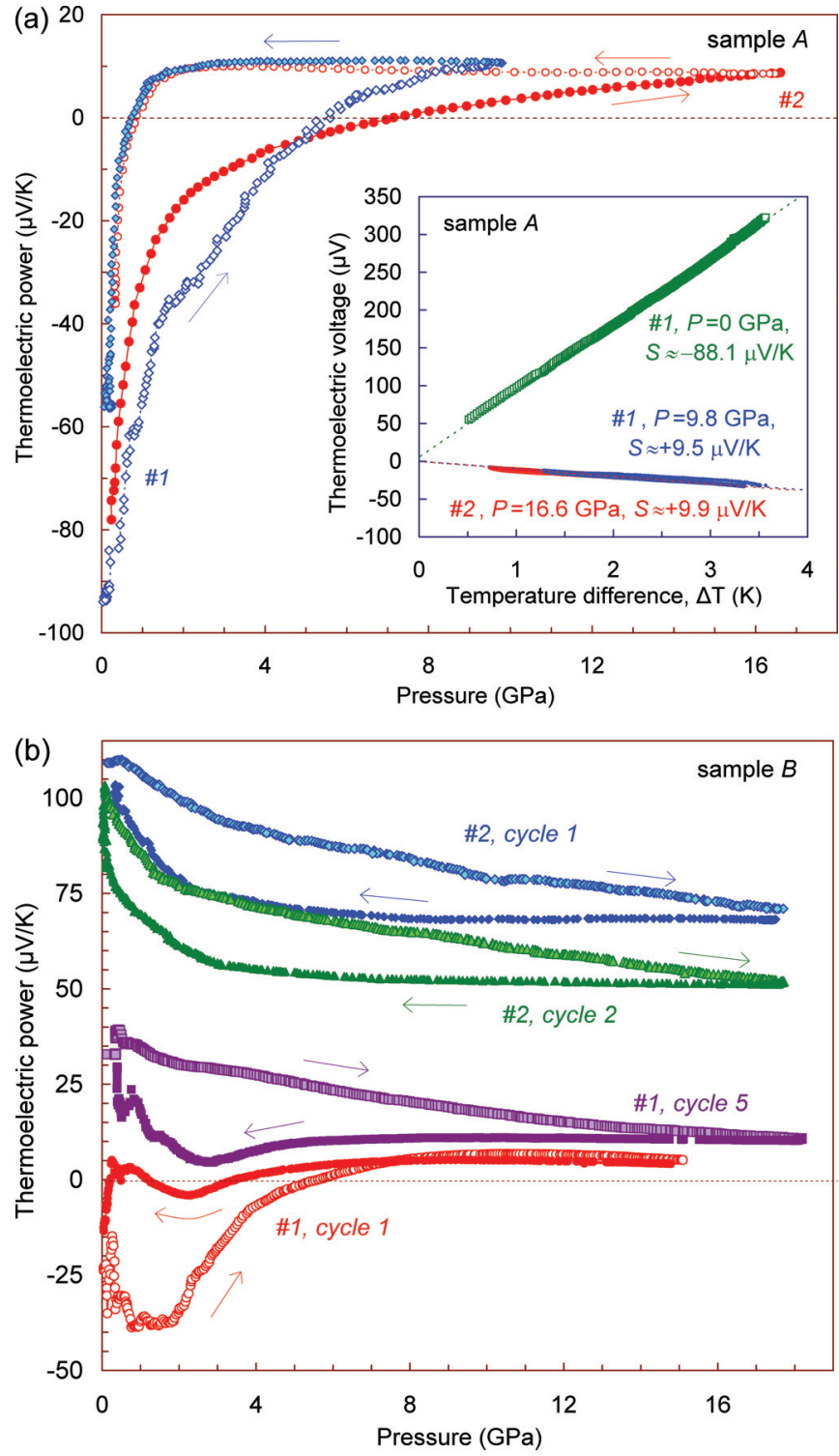

FIG. 7. (Color online) Pressure dependencies of thermoelectric power (Seebeck effect) of samples $A$ (a) and $B$ (b) of the golden $\mathrm{Th}_{2} \mathrm{~S}_{3}$-type $\mathrm{Ti}_{2} \mathrm{O}_{3}$ at ambient temperature. Labels \#1 and \#2 mark two different samples cut from the same bulk samples $A$ and $B$. Arrows show directions of pressure variation. (a) These thermopower curves were measured in a high-pressure cell with both semispherically concave (\#1) and conventional flat anvils (\#2; Ref. 52). Sign inversion of the thermopower from negative to positive above 6-8 GPa unambiguously suggests the dominant conductivity-type inversion from $n$ to $p$ type. Inset shows examples of thermopower determination from linear slopes of dependencies of thermoelectric voltage on temperature difference, $\Delta T$ along the sample thickness. (b) These thermopower curves were measured in a high-pressure cell with conventional flat anvils. Sample $B$ has been established earlier to have regions of dominant $n$ - and $p$-type conductivity (Ref. 1). Thus, plot (b) presents pressure dependencies of thermopower of $n$ - and p-type samples (\#1 and \#2, respectively). Sample \#1 with original $n$-type conductivity behaves similarly to both samples $A$ (a).

dependencies of the electrical resistivity under pressure up to $21 \mathrm{GPa}$ (Figs. 9-11). For these studies, we selected several samples cut from the two bulk ingot synthesized at HP-HT 


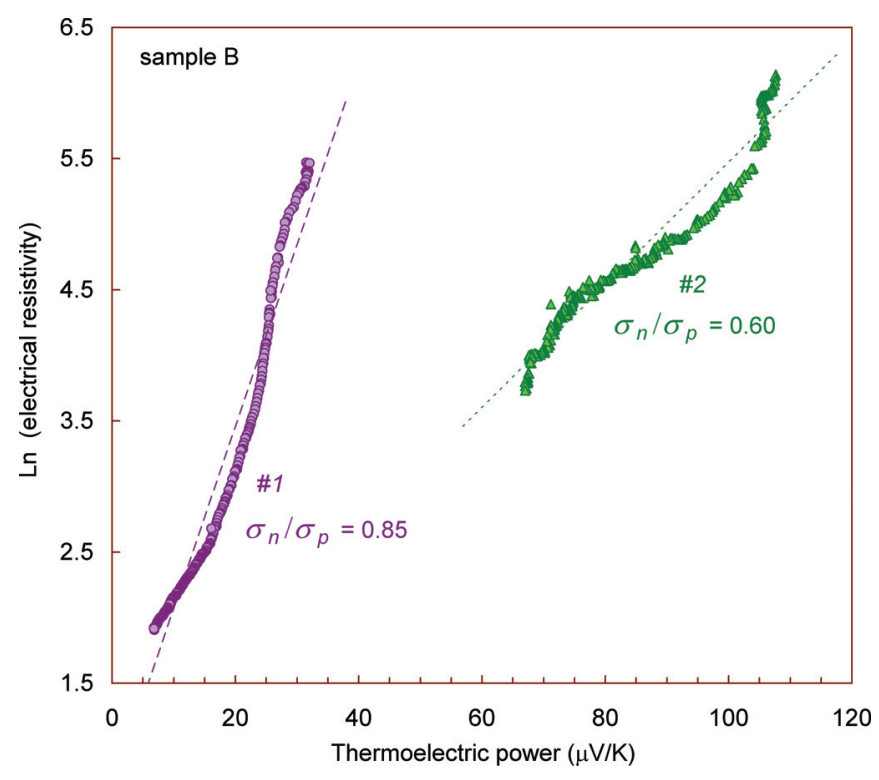

FIG. 8. (Color online) Parametric electrical resistivity vs thermopower dependencies for samples \#1 and \#2 cut from the same bulk sample $B$ of the golden $\mathrm{Th}_{2} \mathrm{~S}_{3}$-type $\mathrm{Ti}_{2} \mathrm{O}_{3}$. These curves were calculated for the second/third pressurization cycles for sample \#2 and for the fourth/fifth pressurization cycles for sample \#1. The $\sigma_{n} / \sigma_{p}$ ratio (parameter $b$ ) may be found from the linear slopes [see Eq. (4) and the following discussion].

conditions (specimens $A$ and $B$; Fig. 6). Ambient pressure investigations of these bulk specimens $A$ and $B$ were reported in Ref. 1.

At ambient conditions, the electrical resistivity values of the golden $\mathrm{Ti}_{2} \mathrm{O}_{3}$ are about $\sim 0.2-0.5 \Omega \times \mathrm{cm}$ (left inset in Fig. 6). With pressure application, the electrical conductivity is apparently enhanced but keeps the values typical for narrow band gap semiconductors [Figs. 6 and 9(a)]. All the samples cut from the bulk specimen $A$ exhibited the dominant
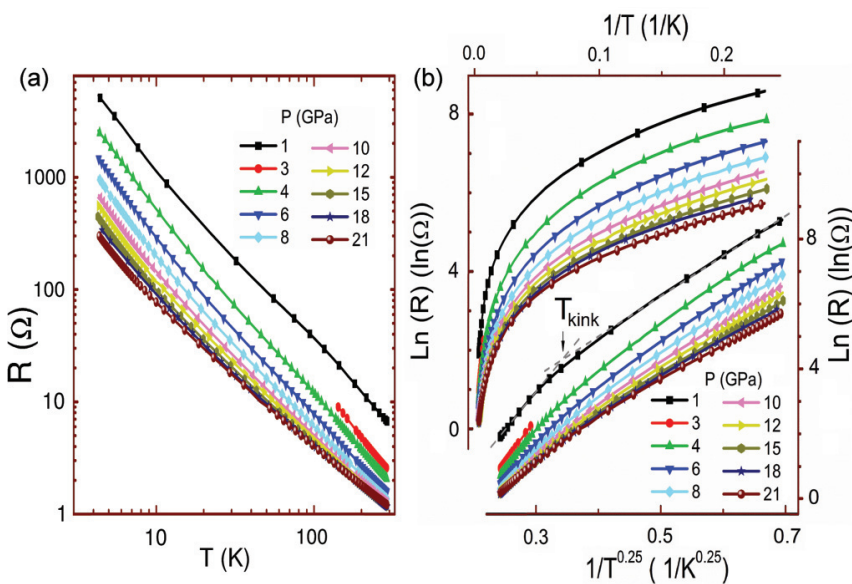

FIG. 9. (Color online) Temperature dependencies of electrical resistance of the golden $\mathrm{Th}_{2} \mathrm{~S}_{3}$-type $\mathrm{Ti}_{2} \mathrm{O}_{3}$ for different applied pressures up to $21 \mathrm{GPa}$. The pressure values are shown at the plots. These temperature dependencies are shown in three representations: (a) in conventional coordinates and (b) in logarithmic electrical resistance vs $1 / T$ (left and top axes) and vs $1 / T^{0.25}$ (right and bottom axes).

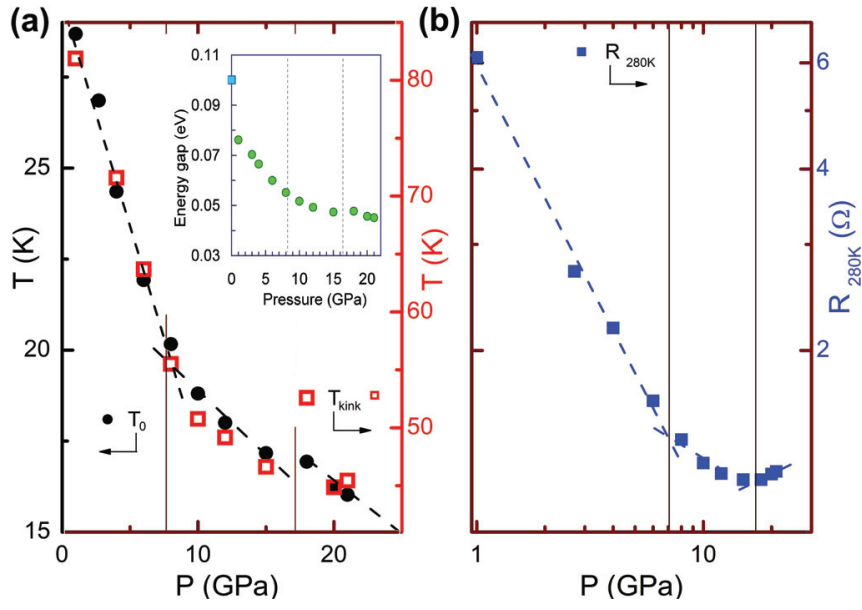

FIG. 10. (Color online) Pressure dependencies of the activation energy $T_{0}$ (left axis) of hopping conductance and $T_{\text {kink }}$ (a) and of electrical resistance at $280 \mathrm{~K}$ (b) of the golden $\mathrm{Th}_{2} \mathrm{~S}_{3}$-type $\mathrm{Ti}_{2} \mathrm{O}_{3}$. Dashed lines represent linear fits of the data in different regions. Inset in (a) shows pressure dependence of the band gap, $E_{g}$, estimated from the activation energies. Ambient value of $E_{g}$ for sample $B$ was taken from Fig. 6. The vertical lines at about 7 and $17 \mathrm{GPa}$ separate different pressure regions on the curves.

n-type conductivity at ambient pressure [Fig. 7(a)]. Applied pressure dramatically reduces the absolute thermopower value [Fig. 7(a)], consistent with the electrical resistivity behavior (Fig. 6). Above 5-7 GPa, the Seebeck coefficient inverts its sign [Fig. 7(a)], which unambiguously suggests a change in the dominant conductivity type from $n$ to $p$ type.

In previous ambient pressure studies, specimen $B$ was found to have an inhomogeneous domain structure with extended regions of both $n$ - and $p$-type conductivities. ${ }^{1}$ Samples cut from specimen $B$ having the dominating $n$-type electrical conductivity [e.g., sample \#1 in Fig. 7(b)] under high-pressure application behave similarly to those of the $n$-type samples cut from specimen A [Fig. 7(a)]. However, $p$-type samples cut from specimen $B$ keep the hole type of

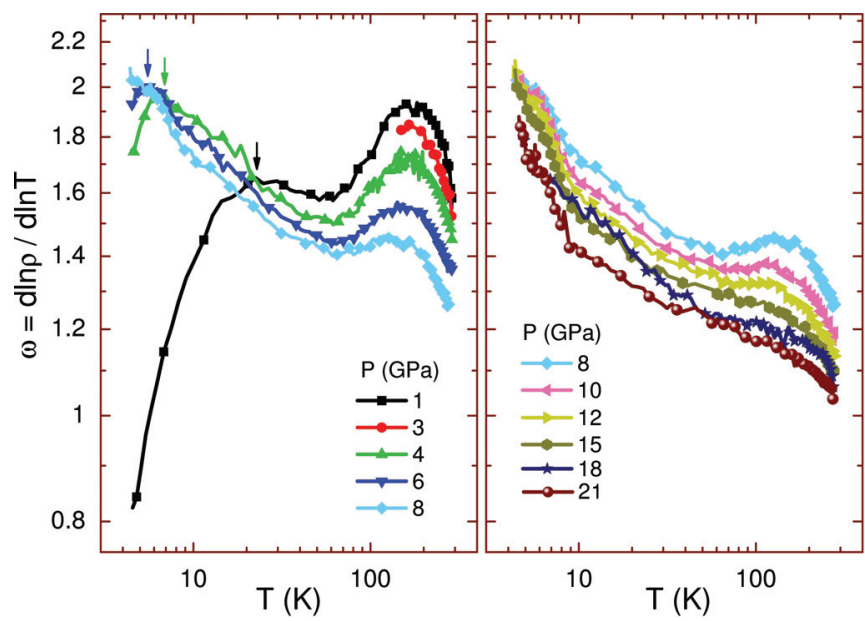

FIG. 11. (Color online) Temperature dependencies of the derivative of the electrical resistance (i.e., $\omega=d \ln \rho / d \ln T$ ) curves from Fig. 9(a) of the golden $\mathrm{Th}_{2} \mathrm{~S}_{3}$-type $\mathrm{Ti}_{2} \mathrm{O}_{3}$ for different applied pressures up to $21 \mathrm{GPa}$. 
conductivity in the entire pressure range investigated, i.e., at least up to $18 \mathrm{GPa}$ [e.g., sample \#2 in Fig. 7(b)]. The thermopower curves of all the measured samples of both $n$ and $p$ types of conductivity demonstrate a smooth slope change at about 2-3 GPa (Fig. 7). Above 6-8 GPa, the thermopower values in samples \#1 and \#2 cut from specimen $A$ and in sample \#1 cut from specimen $B$ become comparatively small, $S \sim+10 \mu \mathrm{V} / \mathrm{K}$, and pressure independent (Fig. 7). Such a thermopower behavior is typical for ultranarrow band gap or strongly compensated semiconductors as well as for semimetals. Notice that a metal state in the $\mathrm{Th}_{2} \mathrm{~S}_{3}$-type $\mathrm{Ti}_{2} \mathrm{O}_{3}$ is excluded based on the electrical resistivity data [Figs. 6 and $9(\mathrm{a})$ ]. It is interesting to notice a similarity between the thermopower behavior under pressure in the golden $\mathrm{Ti}_{2} \mathrm{O}_{3}$ [Fig. 7(a)] and SmS. ${ }^{89}$ In $\mathrm{SmS}$, the pressure-induced phase transition from the ambient black phase, with $E_{g} \sim 0.1 \mathrm{eV}$, to the golden phase, with a pseudogap of about $0.01 \mathrm{eV}, 82,83$ was accompanied by resembling pressure-driven changes in the thermopower from $-90 \mu \mathrm{V} / \mathrm{K}$ at ambient pressure to $+20 \mu \mathrm{V} / \mathrm{K}$ above $\sim 1 \mathrm{GPa} .{ }^{89}$

Although the pressure-driven variations in the thermoelectric properties of the samples are basically reversible, one can still notice that the pressure cycling stimulates the $p$-type conductivity (Fig. 7) and can even irreversibly invert the conductivity type [e.g., in sample \#1 in Fig. 7(b)]. As these samples of the golden $\mathrm{Ti}_{2} \mathrm{O}_{3}$ were found to be intrinsic semiconductors with carrier concentrations of about $10^{17}$ $10^{18} \mathrm{~cm}^{-3}$ (Ref. 1), their electronic properties may be sensitive to defects. This might suggest that the pressure treatment of the golden $\mathrm{Ti}_{2} \mathrm{O}_{3}$ can lead to formation of electrically active defects of acceptor type, thereby changing the balance of $n$ - and $p$-type contributions to the total conductivity. In order to analyze the pressure-driven changes in the electronic transport properties, we used the known expression for the thermoelectric power $(S)$ of nearly intrinsic semiconductor with two-band conductivity, as follows: ${ }^{50,59,90}$

$$
\begin{aligned}
S= & -\frac{k}{|e|}\left[\frac{b-1}{b+1} \cdot \frac{E_{g}}{2 k T}+\left(r_{n}+\frac{5}{2}\right) \frac{b}{b+1}\right. \\
& \left.-\left(r_{p}+\frac{5}{2}\right) \frac{1}{b+1}-\frac{3}{4} \ln \frac{m_{p}^{*}}{m_{n}^{*}}\right],
\end{aligned}
$$

where $k$ is Boltzmann's constant, $e$ is the electron charge $(k / e \approx 86.4 \mu \mathrm{V} / \mathrm{K}), b=\sigma_{n} / \sigma_{p}$ is the ratio of partial conductivities of electrons and holes, $T$ is the temperature, and $r_{n}\left(r_{p}\right)$ and $m_{n}^{*}\left(m_{p}^{*}\right)$ are the scattering parameter and the effective mass of density of states of electrons (holes), respectively.

Using Eq. (4), one can roughly estimate the $\sigma_{n} / \sigma_{p}$ ratio for the known $E_{g}$ and $S$ values. For instance, assuming the dominating acoustic phonon-scattering mechanism of charge carriers, which is typical for intrinsic semiconductors (i.e., assuming $r_{n}=r_{p}=\frac{1}{2}$ ), and neglecting the last logarithmic term, for specimen $A$ with $S \sim-90 \mu \mathrm{V} / \mathrm{K}$ [Fig. 7(a)] and $E_{g}=0.2 \mathrm{eV}$ (right inset in Fig. 6), we can find that at ambient pressure the $\sigma_{n} / \sigma_{p}$ ratio is about $\sim 1.35$. Likewise, for sample \#2 of specimen $B$ with $S \sim+110 \mu \mathrm{V} / \mathrm{K}$ [Fig. 7(b)] and $E_{g}=$ $0.1 \mathrm{eV}$ (right inset in Fig. 6), we can find the $\sigma_{n} / \sigma_{p} \sim 0.6$. In intrinsic semiconductors, $E_{g}$ is directly related to the electrical resistivity $\rho\left(\rho=\rho_{0} \exp \left[E_{g} /(2 k T)\right]\right) .{ }^{50,59,90}$ Equation (4) is a linear function of $E_{g}$, and, using the above expression, one can find a relation between $S$ and $\rho$ as follows: $S \approx-(k / e)[(b-$ $\left.1) /(b+1)]\left(\ln \rho-\ln \rho_{0}\right)-\frac{3}{4}\left(\ln \left(m_{p} / m_{n}\right)\right)\right]$. Since the pressure dependence of $\rho_{0}$ is much weaker than $\rho$, the former could be neglected. From this expression, one can estimate the $\sigma_{n} / \sigma_{p}$ ratio (parameter $b$ ).

The thermopower behavior of the $n$-type samples under pressure (Fig. 7) indicates a strong variation in the $\sigma_{n} / \sigma_{p}$ ratio; hence, these data do not allow us to figure out a contribution of the band gap variation to the thermopower [Eq. (4)]. On the contrary, the moderate variation in the thermopower of the $p$-type sample under pressure [Fig. 7(b)] suggests that the band gap tuning might give a major contribution to the thermopower behavior. In Fig. 8, we show two examples of parametric $\ln R$ vs $S$ curves for samples \#1 and \#2 of specimen $B$. Sample \#2 keeps the $p$-type conductivity across the all pressure cycles, and we find that an average slope of its $\ln R$ vs $S$ curve corresponds to the $\sigma_{n} / \sigma_{p} \sim 0.6$ (Fig. 8). Sample $\# 1$ of specimen $B$ irreversibly inverts the Seebeck coefficient sign from negative to positive after the second pressure run up to $16 \mathrm{GPa}$, and for the fifth thermopower cycle we find that its average ratio $\sigma_{n} / \sigma_{p}=0.85$ is still higher than the one in sample \#2 (Fig. 8). However, one can see that the $\ln R$ vs $S$ curves are apparently nonlinear (Fig. 8); hence, the partial electron and hole conductivities in these samples gradually alter under pressure. The thermopower behavior of sample \#2 of specimen $B$ indicates that its band gap decreases roughly twofold to 6-8 GPa [Fig. 7(b)], thereby suggesting a transition to an ultranarrow band gap state.

The low-temperature electrical resistivity studies performed on bulk sample cut from specimen $B$ directly confirm the persistence of the semiconducting properties up to $21 \mathrm{GPa}$ [Fig. 9(a)], in good agreement with the above room-temperature electronic transport data (Figs. 6 and 7). In these studies, we did not find any new low-temperature phases. This is in contrast with the previously mentioned case of the golden $\mathrm{SmS}$ that exhibited a magnetic ordering and a metal state above $2 \mathrm{GPa}$ and below $\sim 30-50 \mathrm{~K}{ }^{91}$

Comparative analysis of the temperature dependencies of the electrical resistance [Fig. 9(a)] using the conventional Arrhenius $(\ln (R)$ vs $1 / T)$ and $\operatorname{Mott}\left(\ln (R)\right.$ vs $\left.1 / T^{1 / 4}\right)$ representations [Fig. 9(b)] shows that the former cannot fit the data in the entire temperature range. The data between ambient temperature and down to $\sim 150 \mathrm{~K}$ can be fitted by the Arrhenius dependence; hence, we can figure out pressure evolutions of the activation energy $E_{a}$ and the band gap $\left(E_{g}=\right.$ $2 E_{a}$ ) [inset in Fig. 10(a)]. The temperature dependencies in the entire range are well described by a Mott's threedimensional (3D) variable range-hopping conductance model $\left[R=R_{0} \exp \left[-\left(T_{0} / T\right)^{1 / d+1}\right]\right.$, where $d$ is the dimensionality of the system, i.e., $\ln (R) \propto 1 / T^{1 / 4}$ for a 3D system; Fig. 9(b)]. In Fig. 9(b), the label $T_{\text {kink }}$ separates two regions with different slopes. A fit of the temperature curves above $T_{\text {kink }}$ (Fig. 9) gives the activation energies, $T_{0}$, of this hopping conductance [Fig. 10(a)]. The data on $T_{\text {kink }}$ and $T_{0}$ together with the resistance at $280 \mathrm{~K}\left(R_{280 \mathrm{~K}}\right)$ point out a crossover near $7 \mathrm{GPa}$ (Fig. 10). This feature has a correspondence with the $n-p$ inversion at about 6-8 GPa, observed by the thermopower (Fig. 7). The lower-pressure regime is characterized by a saturation of the resistivity represented by a maximum at low 
temperature in the derivative $\omega=d \ln \rho / d \ln T$ that decreases as the pressure is increased (Fig. 11). One can also notice that the electrical resistance at $280 \mathrm{~K}$ starts to rise slightly above 15-18 GPa [Fig. 10(b)], and, in addition, the $T_{\text {kink }}$ and $T_{0}$ curves show weak discontinuities at these pressures [Fig. 10(a)].

The features we found in the thermopower (Fig. 7) and in the electrical resistance curves [Figs. 6 and 9(a)] have a correspondence with those in the structural (Figs. 2 and 3 ) and spectroscopic data (Figs. 4 and 5). For instance, the anomaly in the compression behavior below $2.5 \mathrm{GPa}$ [e.g., a feature in the $c / a$ ratio in Fig. 2(b)] corresponds to the slope change in the thermopower curves near 1.5-3 GPa (Fig. 7). The charge disproportionation process between the Ti1 and Ti2 cations that was found to show up above $\sim 5-9$ GPa [Fig. 3(e)] is compatible with the enhanced electrical conductivity above 6-8 GPa [Figs. 6 and 9(a)], as well as with the $n-p$ inversion at similar pressures (Fig. 7). Charge disproportionation reactions under pressure between cations in other oxides, e.g., in $\mathrm{BiNiO}_{3}$ (Ref. 68) and $\mathrm{LaCu}_{3} \mathrm{Fe}_{4} \mathrm{O}_{12}$ (Ref. 71) were also accompanied by significant rising in the electrical conductivity. One could expect a smooth inflection point in electrical resistivity curve corresponding to the extrema at the BVS curves [Fig. 3(e)]. In fact, the resistance curve shown in Fig. 10(b) suggests a saturation or minimum near $16 \mathrm{GPa}$, but, regretfully, electronic transport studies to higher pressures were not possible at the moment.

\section{CONCLUSIONS}

In summary, we have established that upon compression the golden $\mathrm{Th}_{2} \mathrm{~S}_{3}$-type polymorph of $\mathrm{Ti}_{2} \mathrm{O}_{3}$ demonstrates a number of intriguing features. We found that moderate high pressure induces a partial charge disproportionation between the cations occupying the Ti1 and Ti2 sites in the crystal lattice [Fig. 3(e)], and this process is accompanied by both the enhancement of electrical conductivity [Figs. 6 and 9(a)] and the $n-p$ inversion of the dominant conductivity type around 6-8 GPa (Fig. 7). This partial charge disproportionation was found to lead to strong structural anomalies in the chemical bonds [Figs. 3(a) and 3(b)] and in the Ti1 and Ti2 polyhedra [Fig. 3(d)]. The Raman spectroscopy studies also showed that the crystal structure undergoes minor structural distortions [Figs. 4(a) and 5(a)]. The electronic transport investigations confirmed that the golden $\mathrm{Ti}_{2} \mathrm{O}_{3}$ keeps its semiconducting properties up to $21 \mathrm{GPa}$ and documented a pressure dependence of its activation energy (Figs. 6, 9, and 10).

We have distinctly observed an isostructural phase transition at $38.5 \mathrm{GPa}$ with a volume drop by $\sim 0.7 \%$ (Fig. 2). We have proposed that this isostructural transition could be related to the $s \rightarrow d$ electron transfer in the Ti atoms. Such a $s \rightarrow d$ electron transfer was predicted to occur under high pressure in the Group IV elements such as $\mathrm{Ti}, \mathrm{Zr}$, and $\mathrm{Hf}^{74}$ Zirconium showed an isostructural transition at about $53 \mathrm{GPa}$ with a volume drop by $\sim 1 \%{ }^{77,78}$ Recent studies on Ti metal documented a jump in the $c / a$ ratio around $36-44 \mathrm{GPa}$, also suggesting an electronic transition. ${ }^{73}$ Recalling that the golden $\mathrm{Ti}_{2} \mathrm{O}_{3}$ is a very dense high-pressure polymorph, ${ }^{1,40,41}$ this might be one of the reasons why this subtle electronic transition in the Ti atoms is well observable in the golden $\mathrm{Ti}_{2} \mathrm{O}_{3}$. Since the $\mathrm{Th}_{2} \mathrm{~S}_{3}$-type $\mathrm{Ti}_{2} \mathrm{O}_{3}$ may be considered as a window to strongly compressed sesquioxides, the above findings in the golden $\mathrm{Ti}_{2} \mathrm{O}_{3}$ hint at fascinating physics in sesquioxides in extreme conditions of high pressure.

\section{ACKNOWLEDGMENTS}

The authors thank M. Hanfland (ESRF) for help in the XRD experiments at ESRF and A.V. Kurnosov (BGI) and D.M. Trots (BGI) for loading the high-pressure cells with gas. SVO also thanks Deutsche Forschungsgemeinschaft (Projects No. OV-110/1-1 and No. OV-110/1-2) for the financial support. A part of this work was performed under financial support of the RFBR (Grant No. 10-08-00945).
*Corresponding author: sergey.ovsyannikov@uni-bayreuth.de

${ }^{1}$ S. V. Ovsyannikov, X. Wu, A. E. Karkin, V. V. Shchennikov, and G. M. Manthilake, Phys Rev. B 86, 024106 (2012), and references therein.

${ }^{2}$ M. Douin, L. Guerlou-Demourgues, M. Menetrier, E. Bekaert, L. Goubault, P. Bernard, and C. Delmas, J. Solid State Chem. 182, 1273 (2009).

${ }^{3}$ M. Jansen and N. Korber, Zeitschrift fuer Anorganische und Allgemeine Chemie 598, 163 (1991).

${ }^{4}$ C. Svensson, Acta Cryst. B 31, 2016 (1975).

${ }^{5}$ S. Geller, J. Chem. Phys. 33, 676 (1960).

${ }^{6} \mathrm{H}$. A. Harwig, Zeitschrift fuer Anorganische und Allgemeine Chemie 444, 151 (1978).

${ }^{7}$ P. S. Aggarwal and A. Goswami, J. Phys. Chem. 65, 2105 (1961). ${ }^{8}$ N. Funamori and R. Jeanloz, Science 278, 1109 (1997).

${ }^{9}$ J. F. Lin, O. Degtyareva, C. T. Prewitt, P. Dera, N. Sata, E. Gregoryanz, H. K. Mao, and R. J. Hemley, Nat. Mater. 3, 390 (2004).
${ }^{10}$ G. K. Rozenberg, L. S. Dubrovinsky, M. P. Pasternak, O. Naaman, T. L. Bihan, and R. Ahuja, Phys. Rev. B 65, 064112 (2002).

${ }^{11}$ E. Ito, H. Fukui, T. Katsura, D. Yamazaki, T. Yoshino, Y. Aizawa, A. Kubo, S. Yokoshi, K. Kawabe, S. Zhai, A. Shatzkiy, M. Okube, A. Nozawa, and K. Funakoshi, Am. Mineral. 94, 205 (2009).

${ }^{12}$ L. Dubrovinsky, T. Boffa-Ballaran, K. Glazyrin, A. Kurnosov, D. Frost, M. Merlini, M. Hanfland, V. B. Prakapenka, P. Schouwink, T. Pippinger, and N. Dubrovinskaia, High Press. Res. 30, 620 (2010).

${ }^{13}$ M. F. Bekheet, M. R. Schwarz, S. Lauterbach, H.-J. Kleebe, P. Kroll, R. Riedel, and A. Gurlo, Angew. Chem., Int. Ed. 52, 6531 (2013).

${ }^{14}$ S. V. Ovsyannikov, D. M. Trots, A. V. Kurnosov, W. Morgenroth, H.-P. Liermann, and L. Dubrovinsky, J. Phys.: Condens. Matter 25, 385401 (2013).

${ }^{15}$ J. Kato, K. Hirose, H. Ozawa, and Y. Ohishi, Am. Mineral. 98, 335 (2013).

${ }^{16}$ B. Xu, H. Stokes, and J. Dong, J. Phys.: Condens. Matter 22, 315403 (2010). 
${ }^{17}$ S. Ono, T. Kikegawa, and Y. Ohishi, J. Phys. Chem. Solids 65, 1527 (2004).

${ }^{18}$ S. Ono and Y. Ohishi, J. Phys. Chem. Solids 66, 1714 (2005).

${ }^{19}$ S. Ono, K. Funakoshi, Y. Ohishi, and E. Takahashi, J. Phys.: Condens. Matter 17, 269 (2005).

${ }^{20}$ A. R. Oganov and S. Ono, Proc. Nat. Acad. Sci. 102, 10828 (2005).

${ }^{21}$ S. Ono, A. R. Oganov, T. Koyama, and H. Shimizu, Earth Planet Sci. Lett. 246, 326 (2006).

${ }^{22}$ J. Santillan, S.-H. Shim, G. Shen, and V. B. Prakapenka, Geophys. Res. Lett. 33, L15307 (2006).

${ }^{23}$ M. Murakami, K. Hirose, K. Kawamura, N. Sata, and Y. Ohishi, Science 304, 855 (2004).

${ }^{24}$ A. R. Oganov and S. Ono, Nature 430, 445 (2004).

${ }^{25}$ A. Gurlo, D. Dzivenko, P. Kroll, and R. Riedel, Phys. Status SolidiRRL 2, 269 (2008).

${ }^{26}$ A. Gurlo, P. Kroll, and R. Riedel, Chem. Eur. J. 14, 3306 (2008).

${ }^{27}$ D. Liu, W. W. Lei, B. Zou, S. D. Yu, J. Hao, K. Wang, B. B. Liu, Q. L. Cui, and G. T. Zou, J. Appl. Phys. 104, 083506 (2008).

${ }^{28}$ H. Yusa, T. Tsuchiya, N. Sata, and Y. Ohishi, Phys. Rev. B 77, 064107 (2008).

${ }^{29}$ A. Gurlo, Angew. Chem. Int. Ed. 49, 5610 (2010).

${ }^{30}$ S. V. Ovsyannikov and L. Dubrovinsky, High Press. Res. 31, 23 (2011).

${ }^{31}$ S. V. Ovsyannikov, A. M. Abakumov, A. A. Tsirlin, W. Schnelle, R. Egoavil, J. Verbeeck, G. Van Tendeloo, K. Glazyrin, M. Hanfland, and L. Dubrovinsky, Angew. Chem. Int. Ed. 52, 1494 (2013).

${ }^{32}$ A. A. Belik, T. Furubayashi, Y. Matsushita, M. Tanaka, S. Hishita, and E. Takayama-Muromachi, Angew. Chem. Int. Ed. 48, 6117 (2009).

${ }^{33}$ A. A. Belik, Y. Matsushita, M. Tanaka, and E. TakayamaMuromachi, Angew. Chem. Int. Ed. 49, 7723 (2010).

${ }^{34}$ H. Yusa, T. Tsuchiya, J. Tsuchiya, N. Sata, and Y. Ohishi, Phys. Rev. B 78, 092107 (2008).

${ }^{35}$ H. Yusa, T. Tsuchiya, N. Sata, and Y. Ohishi, Inorg. Chem. 48, 7537 (2009).

${ }^{36}$ H. Yusa, T. Tsuchiya, N. Sata, and Y. Ohishi, Inorg. Chem. 49, 4478 (2010).

${ }^{37}$ K. Umemoto and R. M. Wentzcovitch, Phys. Chem. Minerals 38, 387 (2011).

${ }^{38}$ S. Ghedia, T. Locherer, R. Dinnebier, D. L. V. K. Prasad, U. Wedig, M. Jansen, and A. Senyshyn, Phys. Rev. B 82, 024106 (2010).

${ }^{39}$ W. H. Zachariasen, Acta. Crystallogr. 2, 321 (1949).

${ }^{40}$ D. Nishio-Hamane, M. Katagiri, K. Niwa, A. Sano-Furukawa, T. Okada, and T. Yagi, High Press. Res. 29, 379 (2009).

${ }^{41}$ S. V. Ovsyannikov, X. Wu, V. V. Shchennikov, A. E. Karkin, N. Dubrovinskaia, G. Garbarino, and L. Dubrovinsky, J. Phys.: Condens. Matter 22, 375402 (2010).

${ }^{42}$ C. Ma and G. R. Rossman, Am. Mineral. 94, 841 (2009).

${ }^{43} \mathrm{~K}$. Umemoto and R. M. Wentzcovitch, Proc. Natl. Acad. Sci. USA 105, 6526 (2008).

${ }^{44}$ Q. Zhang, X. Wu, and S. Qin, Eur. Phys. J. B 85, 267 (2012).

${ }^{45}$ D. J. Frost, B. T. Poe, R. G. Trønnes, C. Liebske, A. Duba, and D. C. Rubie, Phys. Earth Planet. Inter. 143-144, 507 (2004).

${ }^{46}$ A. Kurnosov, I. Kantor, T. Boffa-Ballaran, S. Lindhardt, L. Dubrovinsky, A. Kuznetsov, and B. H. Zehnder, Rev. Sci. Instrum. 79, 045110 (2008).

${ }^{47}$ H. K. Mao, J. Xu, and P. M. Bell, J. Geophys. Res. 91, 4673 (1986).

${ }^{48}$ A. P. Hammersley, S. O. Svensson, M. Hanfland, A. N. Fitch, and D. Hausermann, High Press. Res. 14, 235 (1996).
${ }^{49}$ J. Rodriguez-Carvajal, Physica B 192, 55 (1993).

${ }^{50}$ S. V. Ovsyannikov, I. V. Korobeinikov, N. V. Morozova, A. Misiuk, N. V. Abrosimov, and V. V. Shchennikov, Appl. Phys. Lett. 101, 062107 (2012)

${ }^{51}$ S. V. Ovsyannikov, H. Gou, N. V. Morozova, I. Tyagur, Y. Tyagur, and V. V. Shchennikov, J. Appl. Phys. 113, 013511 (2013).

${ }^{52}$ V. V. Shchennikov, S. V. Ovsyannikov, and A. Y. Manakov, J. Phys. Chem. Solids 71, 1168 (2010).

${ }^{53}$ S. V. Ovsyannikov, V. V. Shchennikov, G. V. Vorontsov, A. Y. Manakov, A. Y. Likhacheva, and V. A. Kulbachinskii, J. Appl. Phys. 104, 053713 (2008).

${ }^{54}$ S. V. Ovsyannikov, V. V. Shchennikov, M. A. Shvetsova, L. S. Dubrovinsky, and A. Polian, Phys. Rev. B 81, 060101 (2010).

${ }^{55}$ S. V. Ovsyannikov and V. V. Shchennikov, Appl. Phys. Lett. 90, 122103 (2007)

${ }^{56}$ S. V. Ovsyannikov, V. V. Shchennikov, A. E. Karkin, A. Polian, O. Briot, S. Ruffenach, B. Gil, and M. Moret, Appl. Phys. Lett. 97, 032105 (2010)

${ }^{57}$ V. V. Shchennikov, S. V. Ovsyannikov, and A. V. Bazhenov, J. Phys. Chem. Solids 69, 2315 (2008).

${ }^{58}$ S. V. Ovsyannikov, N. V. Morozova, A. E. Karkin, and V. V. Shchennikov, Phys. Rev. B 86, 205131 (2012).

${ }^{59}$ V. V. Shchennikov, N. V. Morozova, I. Tyagur, Y. Tyagur, and S. V. Ovsyannikov, Appl. Phys. Lett. 99, 212104 (2011).

${ }^{60}$ V. V. Shchennikov, N. V. Morozova, and S. V. Ovsyannikov, J. Appl. Phys. 111, 112624 (2012).

${ }^{61}$ G. Garbarino, M. Monteverde, M. Núñez-Regueiro, C. Acha, M. L. Foo, and R. J. Cava, Phys. Rev. B 77, 064105 (2008).

${ }^{62}$ See Supplemental Material at http://link.aps.org/supplemental/ 10.1103/PhysRevB.88.184106 for unit cell parameters of the golden $\mathrm{Ti}_{2} \mathrm{O}_{3}$ under pressure.

${ }^{63}$ D. P. Kozlenko and B. N. Savenko, J. Phys.: Condens. Matter 16, 9031 (2004).

${ }^{64}$ N. E. Brese and M. O'Keffee, Acta Cryst. B 47, 192 (1991).

${ }^{65}$ A. I. Poteryaev, A. I. Lichtenstein, and G. Kotliar, Phys. Rev. Lett. 93, 086401 (2004).

${ }^{66}$ T. Seda and G. R. Hearne, J. Phys. Condens. Matter 16, 2707 (2004).

${ }^{67} \mathrm{X}$. Wu, G. Steinle-Neumann, O. Narygina, I. Kantor, C. McCammon, S. Pascarelli, G. Aquilanti, V. Prakapenka, and L. Dubrovinsky, Phys. Rev. B 79, 094106 (2009).

${ }^{68}$ M. Azuma, S. Carlsson, J. Rodgers, M. G. Tucker, M. Tsujimoto, S. Ishiwata, S. Isoda, Y. Shimakawa, M. Takano, and J. P. Attfield, J. Am. Chem. Soc. 129, 14433 (2007).

${ }^{69}$ L. Bai, M. Pravica, Y. Zhao, C. Park, Y. Meng, S. V. Sinogeikin, and G. Shen, J. Phys.: Condens. Matter 24, 435401 (2012).

${ }^{70}$ Y. W. Long, N. Hayashi, T. Saito, M. Azuma, S. Muranaka, and Y. Shimakawa, Nature 458, 60 (2009).

${ }^{71}$ Y.-W. Long, T. Kawakami, W.-T. Chen, T. Saito, T. Watanuki, Y. Nakakura, Q.-Q. Liu, C.-Q. Jin, and Y. Shimakawa, Chem. Mater. 24, 2235 (2012).

${ }^{72}$ I. Yamada, K. Takata, N. Hayashi, S. Shinohara, M. Azuma, S. Mori, S. Muranaka, Y. Shimakawa, and M. Takano, Angew. Chem. Int. Ed. 47, 7032 (2008).

${ }^{73}$ N. Velisavljevic and G. N. Chesnut, Appl. Phys. Lett. 91, 101906 (2007).

${ }^{74}$ G. Jyoti and S. C. Gupta, J. Phys.: Condens. Matter 6, 10273 (1994) and references within.

${ }^{75}$ H. Xia, G. Parthasarathy, H. Luo, Y. K. Vohra, and A. L. Ruoff, Phys. Rev. B 42, 6736 (1990). 
${ }^{76}$ J. S. Gyanchandani, S. C. Gupta, S. K. Sikka, and R. Chidambaram, J. Phys.: Condens. Matter 2, 6457 (1990).

${ }^{77}$ Y. Akahama, M. Kobayashi, and H. Kawamura, J. Phys. Soc. Jpn. 60, 3211 (1991).

${ }^{78}$ Y. Akahama, M. Kobayashi, and H. Kawamura, High Pres. Res. 10, 711 (1992).

${ }^{79}$ N. Velisavljevic, G. N. Chesnut, L. L. Stevens, and D. M. Dattelbaum, J. Phys.: Condens. Matter 23, 125402 (2011).

${ }^{80}$ A. L. Kutepov and S. G. Kutepova, Phys. Rev. B 67, 132102 (2003).

${ }^{81}$ A. Jayaraman, V. Narayanamurti, E. Bucher, and R. G. Maines, Phys. Rev. Lett. 25, 1430 (1970).

${ }^{82}$ K. Matsubayashi, K. Imura, H. S. Suzuki, G. Chen, N. Mori, T. Nishioka, K. Deguchi, and N. K. Sato, J. Phys. Soc. Jpn. 76, 033602 (2007).

${ }^{83}$ K. Imura, K. Matsubayashi, H. S. Suzuki, N. Kabeya, K. Deguchi, and N. K. Sato, J. Phys. Soc. Jpn. 78, 104602 (2009).

${ }^{84}$ G. K. Rozenberg, M. P. Pasternak, W. M. Xu, L. S. Dubrovinsky, S. Carlson, and R. D. Taylor, Europhys. Lett. 71, 228 (2005).
${ }^{85}$ K. Oka, M. Azuma, W. Chen, H. Yusa, A. A. Belik, E. Takayama-Muromachi, M. Mizumaki, N. Ishimatsu, N. Hiraoka, M. Tsujimoto, M. G. Tucker, J. P. Attfield, and Y. Shimakawa, J. Am. Chem. Soc. 132, 9438 (2010).

${ }^{86}$ J. G. Zhao, L. X. Yang, Y. Yu, S. J. You, R. C. Yu, L. C. Chen, F. Y. Li, C. Q. Jin, X. D. Li, Y. C. Li, and J. Liu, Chin. Phys. Lett. 22, 1199 (2005).

${ }^{87}$ H. Olijnyk, S. Nakano, A. P. Jephcoat, and K. Takemura, Phys. Rev. B 74, 104302 (2006).

${ }^{88}$ V. V. Shchennikov, S. V. Ovsyannikov, and A. Y. Derevskov, Phys. Solid State 44, 1845 (2002).

${ }^{89}$ T. G. Ramesh and V. Shubha, Solid State Commun. 19, 591 (1976).

${ }^{90}$ K. Seeger, Semiconductor Physics (Wien, New York: SpringerVerlag, 1973).

${ }^{91}$ A. Barla, J. P. Sanchez, Y. Haga, G. Lapertot, B. P. Doyle, O. Leupold, R. Ruffer, M. M. Abd-Elmeguid, R. Lengsdorf, and J. Flouquet, Phys. Rev. Lett. 92, 066401 (2004). 